\section{SANDIA REPORT}

SAND97-1766 • UC-403

Unlimited Release

Printed July 1997
RECEIVED

AUG 111997

OSTI

\title{
LDRD Final Report: Physical Simulation of Nonisothermal Multiphase Multicomponent Flow in Porous Media
}

M. J. Martinez, P. L. Hopkins, J. N. Shadid

Prepared by

Sandia National Laboratories

Albuquerque, New Mexico 87185 and Livermore, California 94550

Sandia is a multiprogram laboratory operated by sandia

Corporation, a Lockheed Martin Company, for the United States

Department of Energy under Contract DE-AC04-94AL85000.

Approved for public release; distribution is unlimited.

in Sandia National Laboratories
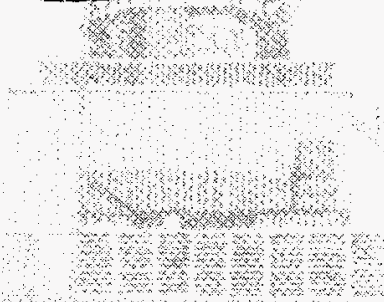

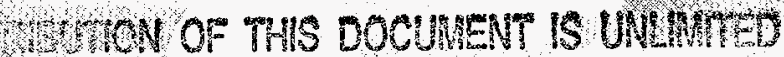
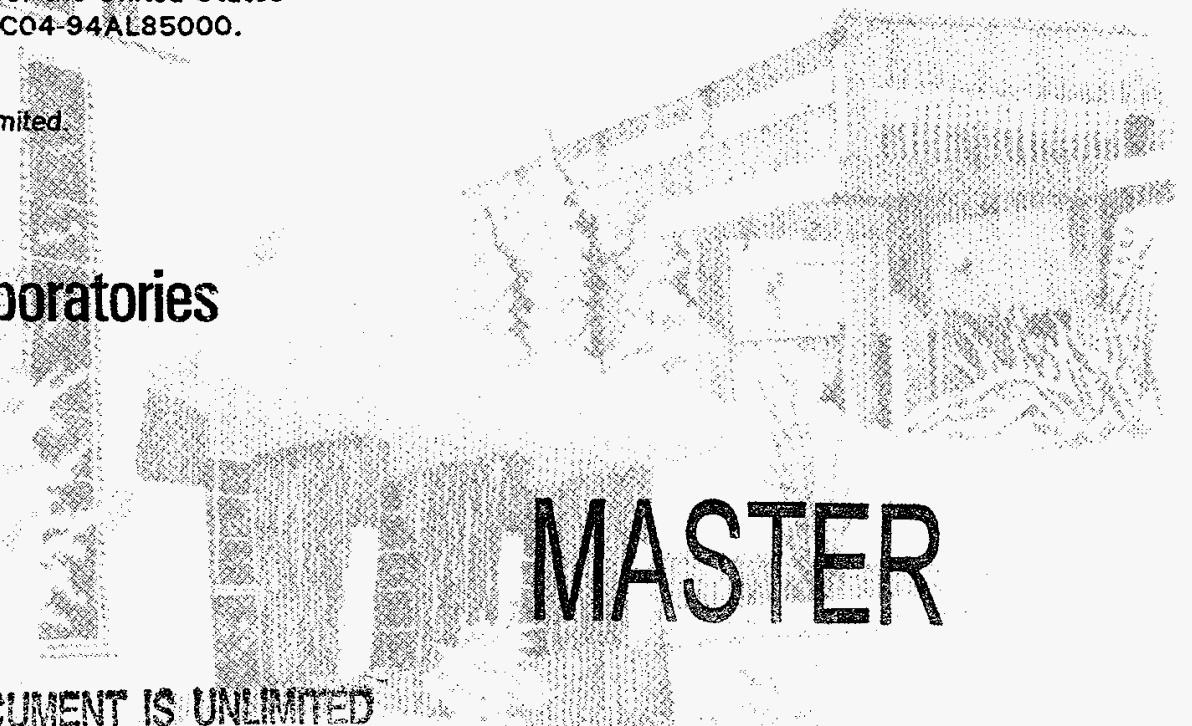

$W^{2}$ 
Issued by Sandia National Laboratories, operated for the United States Department of Energy by Sandia Corporation.

NOTICE: This report was prepared as an account of work sponsored by an agency of the United States Government. Neither the United States Government nor any agency thereof, nor any of their employees, nor any of their contractors, subcontractors, or their employees, makes any warranty, express or implied, or assumes any legal liability or responsibility for the accuracy, completeness, or usefulness of any information, apparatus, product, or process disclosed, or represents that its use would not infringe privately owned rights. Reference herein to any specific commercial product, process, or service by trade name, trademark, manufacturer, or otherwise, does not necessarily constitute or imply its endorsement, recommendation, or favoring by the United States Government, any agency thereof, or any of their contractors or subcontractors. The views and opinions expressed herein do not necessarily state or reflect those of the United States Government, any agency thereof, or any of their contractors.

Printed in the United States of America. This report has been reproduced directly from the best available copy.

Available to DOE and DOE contractors from

Office of Scientific and Technical Information

P.O. Box 62

Oak Ridge, T'N 37831

Prices available from (615) 576-8401, FTS 626-8401

Available to the public from

National Technical Information Service

U.S. Department of Commerce

5285 Port Royal Rd

Springfield, VA 22161

NTIS price codes

Printed copy: A04

Microfiche copy: A01 


\section{DISCLAMIER}

Portions of this docoment may be Illegible in electronic image produets. Images are produced from the best avaitable original doevoment 


\section{DISCLAIMER}

This report was prepared as an account of work sponsored by an agency of the United States Government. Neither the United States Government nor any agency thereof, nor any of their employees, make any warranty, express or implied, or assumes any legal liability or responsibility for the accuracy, completeness, or usefulness of any information, apparatus, product, or process disclosed, or represents that its use would not infringe privately owned rights. Reference herein to any specific commercial product, process, or service by trade name, trademark, manufacturer, or otherwise does not necessarily constitute or imply its endorsement, recommendation, or favoring by the United States Government or any agency thereof. The views and opinions of authors expressed herein do not necessarily state or reflect those of the United States Government or any agency thereof. 


\title{
LDRD Final Report: Physical Simulation of Nonisothermal Multiphase Multicomponent Flow in Porous Media
}

\author{
M. J. Martinez and P. L. Hopkins \\ Engineering Sciences Center \\ J. N. Shadid \\ Computational/Computer Sciences \& Math Center \\ Sandia National Laboratories \\ Albuquerque, NM 87185
}

\begin{abstract}
This document reports on the accomplishments of a laboratory-directed research and development (LDRD) projeci whose objective was to initiate a research program for developing a fundamental understanding of multiphase multicomponent subsurface transport in heterogeneous porous media and to develop parallel processing computational tools for numerical simulation of such problems. The main achievement of this project was the successful development of a general-purpose, unstructured grid, multiphase thermal simulator for subsurface transport in heterogeneous porous media implemented for use on massively parallel (MP) computers via message-passing and domain decomposition iechniques. The numerical platform provides an excellent base for new and continuing project development in areas of current interest to SNL and the DOE complex including, subsurface nuclear waste disposal and cleanup, groundwater availability and contamination studies, fuel-spill transport for accident analysis, and DNAPL transport and remediation.
\end{abstract}




\section{Acknowledgment}

The authors thank C. K. Ho and C. E. Hickox for many helpful discussions. We are especially indebted to R. R. Eaton for providing the code for the ECM material model discussed in section 5. We also wish to thank G. L. Hennigan for much valuable assistance with implementations on the Intel Paragon, and especially to M. W. Glass for assistance with implementations on the Engineering Sciences LAN and with graphical output using the MUSTAFA application.

Sandia is a multiprogram laboratory operated by Sandia Corporation, a Lockheed MartinCompany, for the United States Department of Energy under Contract DE-ACO494AL85000. 


\section{Table of Contents}

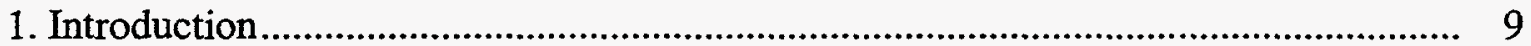

1.1 Summary of Accomplishments .................................................................. $\quad 10$

1.1.1 Mathematical formulation .................................................................... 11

1.1.2 Numerical formulation.................................................................... 11

1.1.3 Numerical Implementation and Verification ................................................ 12

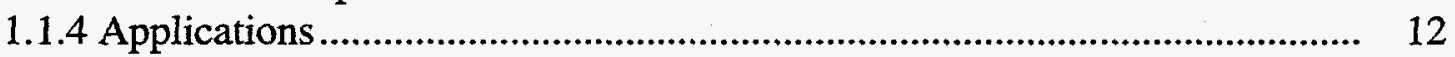

1.1.5 Experimental Program ................................................................................. 13

1.2 Preview of the Report............................................................................... 13

2. Mathematical Formulation ..........................................................................

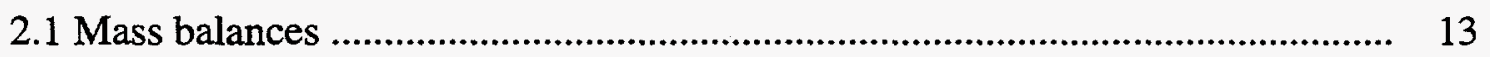

2.2 Energy balance ........................................................................................ 14

3. Constitutive and Thermodynamic Relations .......................................................... 15

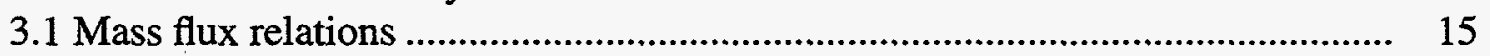

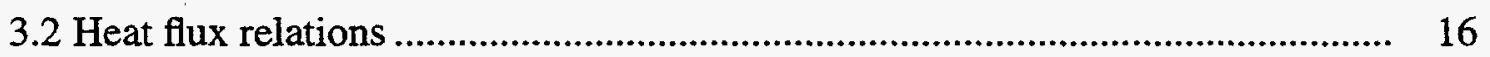

3.3 Thermodynamics............................................................................................ 17

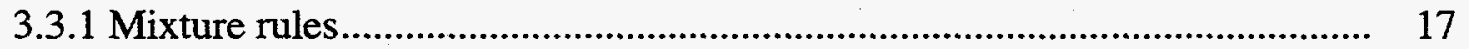

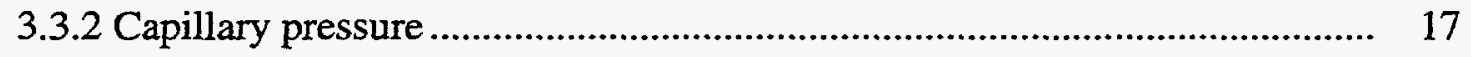

3.3.3 Equations of state....................................................................................... 17

3.4 Transport parameters...................................................................................... 18

3.4.1 Relative permeability functions .................................................................. 18

3.4.2 Binary gas diffusion.............................................................................. 18

3.4.3 Effective thermal conductivity ............................................................. 19

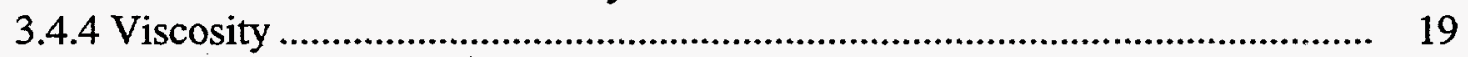

4. Numerical Formulation ............................................................................. 19

4.1 Spatial discretization via the Galerkin Finite Element Method ........................... 20

4.2 Time Integration ......................................................................................... 22

4.2.1 Predictor/corrector methods........................................................................ 22

4.2.2 Time step control ....................................................................................... 23

4.3 Primary Variables .......................................................................................... 24

4.4 Nonlinear and Linear Solution Procedures ......................................................... 25

4.4.1 Inexact Newton iteration........................................................................... 25

4.4.2 Linear systems solvers ................................................................................. 26

4.5 Boundary conditions ..................................................................................... 26

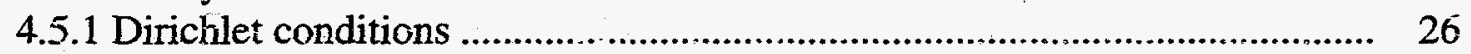

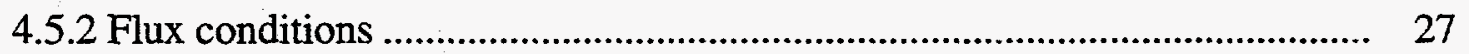

4.6 Parallel Implementation .............................................................................. 27

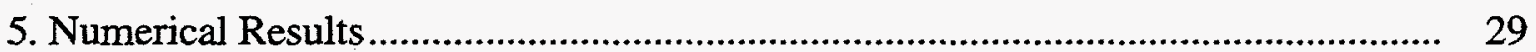

5.1 Benchmark Problems ................................................................................... 29

5.1.1 One-dimensional infiltration into a homogeneous porous material .............. 29

5.1.2 One-dimensional infiltration into a layered medium....................................... 31

5.1.3 One-dimensional heat injection into a homogeneous porous material .......... 33

5.1.4 Three-dimensional flow in unsaturated heterogeneous porous media .......... 35 
5.2 Illustrative Applications ....

5.2.1 Pre-emplacement infiltration at Yucca Mountain...................................... 39

5.2.2 Hydrothermal transport in fractured rock .............................................. 44

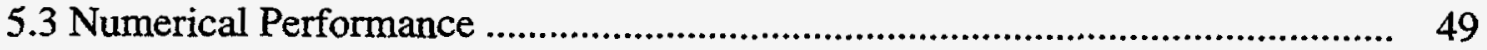

5.3.1 Pre-emplacement infiltration at Yucca Mountain..................................... 49

5.3.2 Hydrothermal transport in fractured rock .............................................. 50

5.3.3 Three-dimensional flow in unsaturated porous media................................. 52

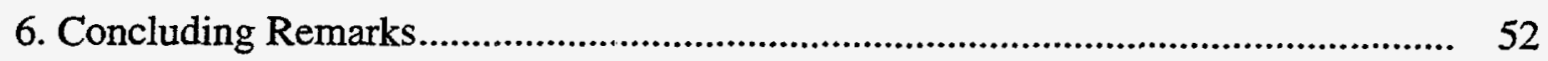

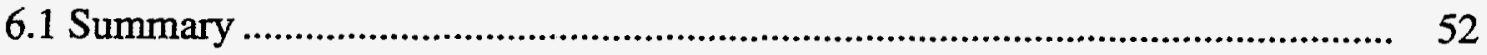

6.2 Recommendations on Future Directions................................................... 53

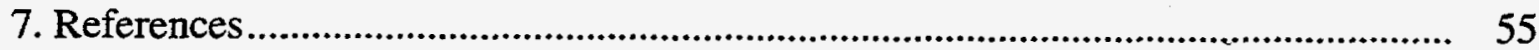




\section{List of Figures}

Figure 1 Schematic of a domain decomposition. ......................................................... 28

Figure 2 Moisture profiles of infiltration into a homogeneous porous material............ 31

Figure 3 Comparison of Cove 2A, case 2 simulation. ............................................... 32

Figure 4 Temperature profiles due to a $20 \mathrm{~W} / \mathrm{m}^{2}$ heat flux (no binary diffusion). .........34

Figure 5 Temperature profiles due to a $20 \mathrm{~W} / \mathrm{m}^{2}$ heat flux (binary diffusion).............. 35

Figure 6 Saturation profiles due to a $100 \mathrm{~W} / \mathrm{m}^{2}$ heat flux (binary diffusion)................ 36

Figure 7 Temperature profiles due to a $100 \mathrm{~W} / \mathrm{m}^{2}$ heat flux (binary diffusion)........... 36

Figure 8 Schematic of two-dimensional problem. ...................................................... 37

Figure 9 Finite element mesh for 3D problem (18,900 brick elements)....................... 38

Figure 10 Saturation contours at $\mathrm{t}=10,20$, and 30 days...............................................40

Figure 11 Comparison of saturation contours at $t=30$ days. ............................................ 41

Figure 12 East-west cross section of Yucca Mountain, Nevada....................................... 42

Figure 13 Liquid saturation distribution (color coded) and flow lines in a cross section of Yucca Mountain, Nevada. ........................................................................... 44

Figure 14 Illustration of a so-called capillary barrier..................................................... 45

Figure 15 Grid composed of 358,000+ node points...................................................... 46

Figure 16 Material blocks and grid on the back side of model...................................... 47

Figure 17 Pressure and temperature distributions at 23 years......................................... 48

Figure 18 Partial air pressure (Pascals) at 23 years in the plane $y=1500 \mathrm{~m}$.................... 49

Figure 19 Parallel processing performance data for the 2D Yucca Mountain pre-emplacement infiltration simulation......................................................................... 50

Figure 20 Parallel processing performance data for the 3D hydrothermal simulation. .. 51

Figure 21 Time step history comparison between the first-order and second order time in-

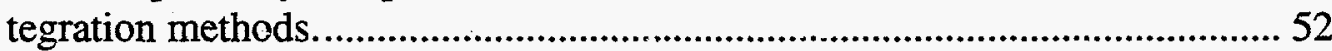

Figure 22 Parallel processing performance data on a network using MPI.....................53 



\section{Introduction}

The objective of this project, funded via the laboratory-directed research and development (LDRD) program at Sandia National Laboratories (SNL), was to develop a fundamental understanding of, and parallel computational tools for simulation of nonisothermal, multiphase, multicomponent flow in heterogeneous porous media. The goal was to develop a "platform" for applying parallel processing capabilities to multiphase subsurface transport. The present document reports on the accomplishments achieved in this project.

Many current activities at SNL require the numerical simulation of multiphase flow in porous media. This capability is critical to on-going SNL activities in environmental remediation, nuclear waste management, reservoir engineering, fuel-spill subsurface transport for accident analysis, and to the assessment of the future availability of groundwater in the $\mathrm{Al}$ buquerque Basin. Furthermore, many new opportunities in related geoscience activities could be pursued if appropriate simulation capabilities were in place. For example, in the area of remediation of hazardous waste disposal sites, one can identify vacuum extraction technologies (including venting as well as thermally-enhanced, resistive and/or radio-frequency heating) and steam injection. In nuclear waste isolation, examples include thermal convection in unsaturated, fractured, geologic media resulting from heat-generating wastes for the Yucca Mountain project. Scientific advancements in each of these areas could benefit from a high-performance numerical simulation capability, the objective of this project.

SNL is investing a large effort in the development of parallel computing and in geoscience technology. The subsurface transport code to be discussed in the following was developed with an architecture that is fully compatible with, and indeed is built around on-going efforts in parallel computing, finite element technology development, and SNL geoscience issues related to current programs in nuclear waste management and environmental remediation. Development of the MP subsurface transport solver leveraged many person-years of effort in the aforementioned computational and geoscience technologies. Against this backdrop. with continued development, the potential exists for a superior mathematical and numerical platform for conducting research into nonisothermal subsurface transport.

There exist a few fine programs which are pursuing similar goals in bringing parallel computing to subsurface transport. The PARFLOW project (e.g., Ashby et al., 1994) at Lawrence Livermore National Laboratory is developing a parallel code which models liquid saturated fow and contaminant transport in heterogeneous media using finite-difference methods on structured, rectangular $(i, j, k)$ grids (Tompson et al., 1996). The Partnership in Computation Sciences (PICS) project (1995 PICS Research Proposal, 1995) is developing a saturated/unsaturated flow and transport model, using the so-called mixed finite eiement method (Raviart and Thomas, 1977), which has also been developed for logically rectangular grids (Arbogast et al., 1994). Similar research projects are being undertaken at the University of Texas Center for Subsurface Modeling under the direction of M. F. Wheeler. The current project has developed subsurface flow and transport models encompassing both different physics and numerical methods. Our finite element architecture allows for fully general unstructured grids. Even though parallel computing brings the ability of unprecedented spatial resolution, structured grid methods are less flexible when 
trying to model complicated geologic features, such as faults, offsets and pinchouts. While the proposal for the PICS project (1995 PICS Research Proposal, 1995) indicates intent to develop an isothermal three-phase flow model, the current project includes mathematical models with energy transport, which is embedded into the two-phase, two-component simulator. Many emerging remediation techniques involve the injection of heat in order to exploit increased volatilities and reduced viscosities helpful to extractions. The energy injection can take the form of heated fluids (e.g. steam) or can be introduced volumetrically, as Joule heating or electromagnetic radiation (e.g. microwave heating). Energy transport is an essential component of a general-purpose subsurface simulator for researching and developing emerging remedial operations.

Given the developing state of subsurface flow modeling using parallel computing, it is essential and advantageous to have more than one group of researchers addressing the problem. Pursuing different approaches to a complex system provides an important means of gaining confidence in the modeling and provides the opportunity to arrive at the optimal solution through consideration of the full range of physical phenomena controlling the system.

The successful completion of this project has raised simulation capabilities for multiphase flow in porous media to a new level; large-scale, 3D multiphase simulations with high resolution of heterogeneities are feasible, and can be performed routinely. The multiphase flow simulator developed on this project not only runs on SNL's MP computers (Intel Paragon, IBM SP-2), but can also be run on networked systems using the MPI message passing software (Gropp et al., 1995). To date mostly 2D subsurface simulations had been possible, due to limitations in simulator capabilities, thereby severely compromising the ability to perform realistic simulations. Simulations performed with our MP code have demonstrated that full $3 \mathrm{D}$, high resolution, simulations of highly heterogeneous geologic media are possible. As a result, we performed a series of high-resolution, pre-emplacement groundwater simulations for the Yucca Mountain project. Such capability enables realistic simulations of subsurface transport in highly heterogeneous media, a needed capability to assess the suitability of Yucca Mountain as the nation's first high-level nuclear waste repository.

\subsection{Summary of Accomplishments}

The major components of the project are enumerated below:

1. Mathematical and numerical formulation

2. Code development

3. Experimental program

In this section we summarize the tasks which were undertaken to meet the goals of this project. 


\subsubsection{Mathematical formulation}

The first task of the project was to develop an appropriate mathematical description of the subject problem, namely nonisothermal, multiphase, multicomponent transport through heterogeneous geologic media. It should be noted that, in contrast to other more established branches of fluid mechanics, a definitive mathematical formulation of nonisothermal multiphase subsurface transport is not well-established. The main problem is that on the pore-scale the governing equations are well-defined, but solving problems on this scale is intractable. Rather, a more practical description is to define variables that are volumeaveraged, and thus represent, on average, the conditions in the porous structure in the vicinity of the considered point. The conjugate problem with averaging is how to define the thermodynamic and transport properties on the scale over which the averaging is performed. A very pertinent example is the capillary pressure. On the pore-scale, the capillary pressure has a definite description as the pressure jump across a curved meniscus between immiscible fluids (liquid and gas, in this work). Capillary pressure is also defined in the averaged equations, now as the difference between the volume-averaged liquid and gas phase pressures. However, it is not clear how to derive a definite (computable) mathematical description of said capillary pressure, as it depends on quantities such as specific interfacial area, which is difficult to define in general (Hassanizadeh and Gray, 1990). Our ultimate strategy was to go with the averaged equations, and to use the literature to arrive at the best available mathematical models for the many thermodynamic and transport parameters required by such an averaged equations approach. Our two-phase and threephase formulations are summarized in Martinez (1995a, 1995b), and in the present report.

\subsubsection{Numerical formulation}

The next task was to develop a numerical formulation suitable for implementing on distributed memory parallel computers. Here there were several important issues to consider. The numerical scheme had to accommodate some special features of nonisothermal, multipase multicomponent flow in porous media. Among these issues was the ability to model phase appearance and disappearance. In the two-phase, two-component model that was implemented, the system may exist in three possible states: 1) all-liquid, 2) all-gas, and 3) liquid and gas. Furthermore, all three states may evolve dynamically in the same computational domain. Moreover, a different set of primary or solution variables can be defined in each state. A technique known as variable-substitution, originally developed in the oil industry (Coats, 1978), is used in some available codes for treating these types of problems. Besides the difficulties foreseen with applying such a method on a parallel computer, another drawback of this method is that it essentially restricts applicable time integration schemes to being first-order. We apply a variable-step predictor-corrector method which has the collateral benefit of providing a rational scheme for time-step selection strategy based on a user-specified local time-truncation error. We researched and developed two persistent variable strategies that circumvent these problems. One involved a flash-calculation-based scheme which pushes the variable-substitution scheme to a phase-equilibrium module. This was implemented into a 1-D finite difference test code for proof-of-concept analysis. We ultimately implemented a slightly modified version of the persistent variable approach, to be presented in the following, which improved on the first scheme. 


\subsubsection{Numerical Implementation and Verification}

The main achievement of this project was the successful development of a general-purpose, unstructured grid, multiphase thermal simulator for subsurface transport in heterogeneous geologic media. A nonisothermal, two-phase (liquid and gas), two-component (water and air) mathematical model was implemented for use on massively parallel (MP) computers via message-passing and domain decomposition techniques, but can also be used on scalar computers. The MPSalsa code (Shadid et al., 1996) was used as a platform for this development effort, as it has served as a focus for the practical application of these SNL developments for parallel processing computers. This LDRD development effort leveraged SNL expertise in domain decomposition (Hendrickson and Leland, 1993), linear solver technology for parallel computers (Hutchinson et al., 1995), finite element technology applied to flow problems and expertise in modeling multiphase flow problems (Martinez 1995a, 1995b; Martinez et al., 1995; Martinez and Eaton, 1996; Martinez and Nilson 1997).

The code has been verified by application to a series of model problems for which (numerical) solutions are available. The simulator has been verified both for isothermal unsaturated flows and, more importantly, for two-phase, heat-driven flows in homogeneous and heterogeneous domains, thus verifying the multiphase implementation. The parallel implementation was also verified by comparing simulations performed on a single processor computer with those performed on parallel computers. These problems have also demonstrated scalability of the MP algorithms. A common misconception is that the parallel capabilities are only accessible on massively parallel computers, but in fact parallel simulations have been performed on a network of workstations, which are fairly common to the scientific community.

\subsubsection{Applications}

Because of its unique capabilities, the code was applied to a high-resolution, fractured flow simulation in cross-sections of Yucca Mountain, Nevada, a location being considered for the nation's first high-level nuclear waste repository. The numerical grid for this cross section contains over 73.000 grid points, with some grid spacings as small as one meter. This is the first flow simulation of Yucca Mountain to include such fine geologic detail, particularly in the Paint Brush geologic unit, which is composed of less fractured rock than the repository horizon, and consequently may act as a buffer zone for groundwater flow and contaminant-laden gas flow (Nilson and Martinez, 1994). The study demonstrated that the moisture flow in the vicinity of the proposed repository horizon can be highly dependent on the stratigraphic units included in the model. To further demonstrate the benefits of MP processing. the code has also been applied to model hydrothermal flow in a large-scale, 3D domann modeled after Yucca Mountain. Pressure, temperature and moisture distributions have been obtained for simulations on a mesh consisting of $358,000+$ grid points in which over 1 million equations are solved at each time step in simulations requiring many 100 s of time steps to simulate flow over many thousands of years. This is a significant capability. 


\subsubsection{Experimental Program}

An experimental program was designed and implemented in tandem with the foregoing tasks (R. R. Eaton ${ }^{1} ;$ R. J. Glass ${ }^{2}$ ). The intent was to develop some archival-quality verification data for heat-driven two-phase flow. Based on preliminary experiments conducted in FY94, the fabrication of the experimental system for conducting multiphase flow experiments was completed in FY96 in the Sandia National Laboratories (SNL) Center 6100 Subsurface Flow Lab. The experiments were designed to measure temperature, saturation, and velocity fields within a forced convective field driven by heating of opposing boundaries. Several side-heated experiments have been performed. Unfortunately, the data indicate insufficient control of heat-losses through the test cell faces, rendering the data unusable as a two-dimensional convection cell, the original intent of the experiment. Because of the heavy heat losses, the convection is three-dimensional. However, the heat loss distribution is unknown, and so the data is not sufficiently characterized for validation, but is useful for understanding two-phase flow phenomenology.

\subsection{Preview of the Report}

The remainder of the report presents the results of the foregoing tasks and activities in more detail. This discussion will serve as the first documentation of the mathematical and numerical model which was developed under this project. The mathematical description of the nonisothermal, two-phase model is presented first. This includes balance equations and all the necessary constitutive and thermodynamic relations which complete the model. Next, the numerical formulation and implementation is discussed. Examples of the application of the code follow, including measures of parallel computing performance.

\section{Mathematical Formulation}

\subsection{Mass balances}

The system under consideration is composed of a porous matrix or skeleton whose interstitial volume is occupied by fluid in motion under various forces. The porosity (interstitial volume fraction) is denoted $\phi$ and is occupied by two phases, liquid and gas. For definiteness, we take the fluid components to be water and air. Each component can partition into each phase. The liquid phase is mostly water, but can have small amounts of air in solution. On the other hand, the gas phase is a mixture of air and water, where each constituent may exist in any fraction between zero and one. In the remainder of this work, subscript $g$ refers to gas, ! to liquid. In order to avoid introducing interphase mass transfer terms, component balances will be developed under the assumption of thermodynamic phase equilibrium.

1. R. R. Eaton, 1994, Pretest calculations for porous flow experiments, SNL memo to R. J. Glass, dtd. April, 15; Investigation of material properties for multiphase laboratory experiments, SNL memo to R. J. Glass, dtd. June 27; Modeling sub-meter scale two-phase laboratory experiments, SNL memo to R. J. Glass, dtd. December 1.

2. R. J. Glass, 1994, Preliminary experimental program for validation of models for nonisothermal multiphase flow in porous media, SNL memo to M. J. Martinez, dtd. March, 20. 
Component balance equations for water, and air, respectively, take the form:

$$
\begin{aligned}
& \frac{\partial d_{w}}{\partial t}+\nabla \cdot F_{w}=Q_{w}, \\
& \frac{\partial d_{a}}{\partial t}+\nabla \bullet F_{a}=Q_{a},
\end{aligned}
$$

where $d_{\alpha}$ is the bulk density of component $\alpha, \boldsymbol{F}_{\alpha}$ denotes the net mass flux vector of component $\alpha$, relative to stationary coordinates, and $Q_{\alpha}$ denotes mass sources. These fluxes embody convective and diffusive transport process, and will be elaborated upon shortly. The bulk densities are given by:

$$
\begin{gathered}
d_{w}=\phi\left(Y_{w l} \rho_{l} S_{l}+Y_{w g} \rho_{g} S_{g}\right), \\
d_{a}=\phi\left(Y_{a l} \rho_{l} S_{l}+Y_{a g} \rho_{g} S_{g}\right),
\end{gathered}
$$

where $Y_{\alpha \beta}$ denotes the mass fraction of component $\alpha$ in phase $\beta, \rho_{\beta}$ are phase densities, which in general are each a mixture of the components, and $S_{\beta}$ are the phase saturations, the fraction of the interstitial volume occupied by phase $\beta$. The pore space is assumed to be fully occupied by fluid,

$$
S_{l}+S_{g}=1 \text {. }
$$

\subsection{Energy balance}

The multiphase system is assumed to be in local thermal equilibrium. In particular, the thermal energy state of the fluids and solid in a representative elementary volume (REV), containing solid, liquid and gas phases, is described by a single average temperature, $T$. An energy balance takes the form

$$
\frac{\partial e}{\partial t}+\nabla \bullet q=Q_{e},
$$

where the bulk internal energy is given by

$$
e=(1-\phi) \rho_{s} e_{s}+\phi\left(S_{l} \rho_{l} e_{l}+S_{g} \rho_{g} e_{g}\right),
$$

where $e_{\beta}$ denotes the specific internal energy of phase $\beta$, and $e_{s}$ is the internal energy of the solid phase. Each phase energy is, in general, dependent on pressure, temperature and composition. Also, $q$ denotes the net heat flux vector, including diffusive and advective transport of heat, and $Q_{e}$ is an extraneous heat source. 


\section{Constitutive and Thermodynamic Relations}

In order to close the system of equations, one must now propose constitutive equations which provide relations between the kinematic variables appearing in the balance equations and the state variables.

\subsection{Mass flux relations}

In describing flow through porous media, one must necessarily pose average quantities (defined with respect to an $\mathrm{REV}$ ) rather than pore-scale continuum values. For example, even though the Darcy fluxes, $v_{\beta}$, have units of velocity, they represent the local volume flux per unit area of porous medium. As such, they cannot satisfy the no-slip condition on fluid/solid interfaces. The flux relations to follow take the place of the momentum balance in continuum equations; they are the average momentum balances under conditions of “creeping flow," i.e., inertia-free, slow viscous flow (e.g., Martinez and Udell, 1990).

The advective fluxes are assumed to be properly described by the extended Darcy law, in which relative permeabilities are introduced to account for the multiphase motion of fluids. Thus the mass fluxes of liquid and gas phases are, respectively,

$$
\begin{gathered}
\rho_{l} v_{l}=f_{l}=-\frac{\rho_{l} k_{r l}}{\mu_{l}} k \cdot\left(\nabla P_{l}+\rho_{l} g\right), \\
\rho_{g} v_{g}=f_{g}=-\frac{\rho_{g} k_{r g}}{\mu_{g}} k \cdot\left(\nabla P_{g}+\rho_{g} g\right),
\end{gathered}
$$

where $P$ is pressure, $g$ is the gravitational acceleration vector, and $\mu$ is dynamic viscosity. Note that we have assumed that each phase has its own phase pressure. The intrinsic permeability tensor of the medium is $k$ and the relative permeabilities are denoted $k_{r \beta}$. The intrinsic permeability tensor is assumed to be a property of the material under consideration, and as such is a spatially heterogeneous quantity.

Each component net mass flux appearing in the balance equations (1), (2) and (6), is assumed to be a superposition of component fluxes in each phase,

$$
F_{\alpha}=F_{\alpha l}+F_{\alpha g},
$$

and each component phase-flux can be written as a sum of an advective (pressure-driven) flux and a diffusive flux,

$$
F_{\alpha \beta}=Y_{\alpha \beta} f_{\beta}+J_{\alpha \beta}
$$

Because the gas is a mixture, each component will undergo interdiffusion whenever a gradient in concentration exists (see, for example, Bird, Stewart and Lightfoot, 1960). The diffusive fluxes in the gas are approximated by

$$
J_{\alpha g}=-\rho_{g} D_{\alpha g} \nabla Y_{\alpha g}
$$


We note here that this form is only strictly valid for binary mixtures. In a multicomponent system with more than two components, the diffusion fluxes appear in the so-called Stefan-Maxwell form (see Appendix E of Williams, 1985) which is an implicit system of equations for the diffusive fluxes in terms of the gradients of mass fraction. Also, Knudsen diffusion is not included in the foregoing diffusion model (but could be easily implemented). Knudsen flow may be significant in porous structures with pore sizes comparable to the mean-free path of the flowing gases (Scheidegger, 1972).

On average, the gas mixture as a whole moves with the average mass flux given by,

$$
f_{g}=F_{w g}+F_{a g} .
$$

Substituting from eqn. (11) into (13), and using the fact that the mass fractions in a phase must sum to unity, the diffusive fluxes in the gas phase must satisfy,

$$
\sum_{\alpha} J_{\alpha g}=0
$$

In general, the diffusive fluxes of components dissolved in the liquid phase could be described in the form

$$
J_{\alpha \beta}=-D_{\alpha \beta} \nabla \rho_{\alpha \beta}, \quad \beta \neq g,
$$

where $\rho_{\alpha \beta}$ is the concentration of component $\alpha$ in phase $\beta$. Diffusion of dissolved air in the liquid phase has been neglected in this implementation.

\subsection{Heat flux relations}

The total heat flux vector includes conductive and convective contributions,

$$
q=-\lambda_{T} \nabla T+\sum_{\beta}\left(\sum_{\alpha} h_{\alpha \beta} F_{\alpha \beta}\right)
$$

where $\lambda_{T}$ is an effective thermal conductivity. The second term on the right-hand-side is the convective flux, and is the sum over all components and phases of the product of the component enthalpy and net flux of component in a particular phase. The phase enthalpies $\left(h_{\beta}\right)$ are also defined via superpositions of component enthalpies in each phase,

$$
\sum_{\alpha} h_{\alpha \beta} F_{\alpha \beta}=\sum_{\alpha} h_{\alpha \beta}\left(Y_{\alpha \beta} f_{\beta}+J_{\alpha \beta}\right)=h_{\beta} f_{\beta}+\sum_{\alpha} h_{\alpha \beta} J_{\alpha \beta}
$$

where $h_{\alpha \beta}$ is the enthalpy of component $\alpha$ as it would exist pure at the same temperature and pressure of the phase $\beta$, and the phase enthalpy is $h_{\beta}=\sum_{\alpha} h_{\alpha \beta} Y_{\alpha \beta}$. For example, $h_{w g}$ is the enthalpy of water vapor at the prevailing temperature and gas pressure. Thus, the convective part of the heat flux vector can be written in the (familiar) form 


$$
q_{c o n v}=\sum_{\beta}\left(\rho_{\beta} v_{\beta} h_{\beta}+\sum_{\alpha} h_{\alpha \beta} J_{\alpha \beta}\right) .
$$

\subsection{Thermodynamics}

\subsubsection{Mixture rules}

Ideal gas mixing rules are used to approximate the thermodynamics of the gaseous phase,

$$
P_{g}=\sum_{\alpha} P_{\alpha g} \quad \rho_{g}=\sum_{\alpha} \rho_{\alpha g}
$$

where $P_{\alpha g}$ and $\rho_{\alpha g}$ are the partial pressure and partial density (concentration) of each component in the gas phase. The mass fractions in each phase must sum to unity,

$$
Y_{w \beta}+Y_{a \beta}=1 \quad \beta=l, g
$$

\subsubsection{Capillary pressure}

Because the transport problem under consideration involves multiple, immiscible phases, capillary forces must be considered. The Darcy flux laws given earlier were expressed with respect to phase pressure gradients. The phase pressures are related via the capillary pressure relation,

$$
P_{g}-P_{l}=P_{c}\left(S_{l}\right)
$$

which, as indicated, are assumed to be empirically specified as a function of the phase saturation of liquid. Several particular models are presented in the section on Numerical Results. We note that the capillary pressure as defined is well motivated on the microscale. Its interpretation on the macroscale is not so easily motivated, and involves postulating the existence of the relations relative to REV-averaged pressures. However, the capillary pressure-saturation relation is commonly assumed to hold for flow in porous media. See Scheidegger (1974), Bear (1972), and Aziz \& Settari (1979) for the "traditional" viewpoint on the role of capillary pressure in porous media. Some more recent discussions can be found in Hassanizadeh and Gray (1990), and references therein.

\subsubsection{Equations of state}

Equations of state must be specified to complete the P-V-T (pressure-volume-temperature) description of the system. For the gas phase, the equations of state take the general form,

$$
\begin{gathered}
\rho_{\alpha g}=\rho_{\alpha g}\left(P_{\alpha g}, T\right) \\
h_{\alpha g}=h_{\alpha g}(T, P)
\end{gathered},
$$

for the concentration (partial density) and enthalpy of component $\alpha$. Notice that the gas phase is approximated as a mixture of ideal gases. The air properties are computed via the 
ideal gas law for a calorically perfect gas. The steam properties are computed using realgas steam table data. The enthalpy of air is independent of pressure under this approximation, while the water vapor enthalpy is pressure and temperature dependent. The energies are found from the definition of the enthalpy, $h_{\alpha g}=e_{\alpha g}+P_{\alpha g} / \rho_{\alpha g}$.

The liquid phase density is computed as a mixture of water with a slight amount of dissolved air. Steam table values are used for the thermodynamic properties of water, in both gas and liquid. The rational polynomial approximations given in Zyvoloski, et al., 1995, are implemented. Because of the low solubility of air in liquid water, a Henry's law partitioning (e.g., Reid et al., 1987) is assumed,

$$
Y_{a l}=\frac{P_{a}}{P_{H, a l}}
$$

where the Henry's "constant", $P_{H, a l}$, has been modified for the molecular weight ratio of water to air.

\subsection{Transport parameters}

\subsubsection{Relative permeability functions}

The measurement of two-phase flow relative permeabilities is a demanding task. Consequently, a variety of methods have been proposed for developing theoretical two-phase relative permeabilities (Aziz and Settari, 1979). See Martinez and McTigue (1991) for a discussion of some commonly invoked models. Each of these models requires a variety of experimentally determined data, such as residual saturations. In general, the two-phase relative permeabilities are specified in the form,

$$
k_{r \beta}=k_{r \beta}\left(S_{\beta}\right)
$$

Notice that in view of the relation in Eq. (5) among the phase saturations, the $\beta$-phase relative permeability is presumed to depend on its own phase saturation. Several particular models are presented in the section on Numerical Results.

\subsubsection{Binary gas diffusion}

The binary diffusion coefficient in free-space depends on temperature and pressure (Reid, et al., 1987). To account for the fact that diffusion is taking place in a porous medium, and to account for the temperature and pressure dependence, the diffusion coefficient is modeled according to (Vargaftik, 1975),

$$
D_{\alpha g}=\frac{\phi S_{g}}{\tau} D_{\alpha g}^{0} \frac{P_{r e f}}{P_{g}}\left(\frac{T}{T_{r e f}}\right)^{v} \text {, }
$$


where $\tau$ is tortuosity and $D_{\alpha g}^{0}$ is the diffusivity in free space at temperature $T_{r e f}$ and pressure $P_{\text {ref }}$. The term $\phi S_{g} / \tau$ modifies the expression for free diffusion to account for the porous skeleton. For air-water systems, $v \approx 2$ is suggested by Pruess (1987).

\subsubsection{Effective thermal conductivity}

The saturation-dependent effective thermal conductivity can be specified as defined by Somerton, et al., (1974), taking the form

$$
\lambda=\lambda_{d r y}+S_{l}^{0}\left(\lambda_{w e t}-\lambda_{d r y}\right),
$$

where $\lambda_{d r y}$ is the conductivity under all-gas conditions, $\lambda_{w e t}$ is the conductivity under water-saturated conditions, and $v$ is nominally in the range [1/2,1], depending on the material type.

\subsubsection{Viscosity}

The gas phase viscosity is computed as

$$
\mu_{g}=\sum_{\alpha} Y_{\alpha g} \mu_{\alpha g}
$$

where $\mu_{\alpha g}$ is the viscosity of component $\alpha$ in the gas phase, generally a strong function of temperature. Because of the low solubility of air in water, the liquid phase viscosity ignores the air content. The temperature-dependent liquid water viscosity is specified according to the rational approximation given by Zyvoloski et al., (1995).

\section{Numerical Formulation}

In this section we discuss our numerical method, based on unstructured grid, finite element techniques, for obtaining approximate solutions to the mathematical model. The flow equations describing the transport of water, air and energy are comprised of the component and energy balances given in the foregoing, which together with equations of constitution and transport parameters, form a closed system of equations. These governing equations constitute a coupled set of highly nonlinear partial differential equations (PDEs).

The numerical method presented for solving the initial-boundary value problem formed by the ccupled system of PDEs is a finite element method (FEM), enabling a general representation of complex geologic stratigraphy. In the present work, the spatial discretization is accomplished by the Galerkin finite element method (e.g. Hughes, 1987). The resulting system of ODEs is integrated forward in time by a variable-step backward-difference predictor-corrector scheme. The backward-difference formulae result in systems of nonlinear algebraic equations to be solved for nodal quantities. The effective treatment of the highly nonlinear system considered here is made possible by the use of Newton iteration. 


\subsection{Spatial discretization via the Galerkin Finite Element Method}

Derivations of the Galerkin finite element method (GFEM) are widely available (e.g., Huebner and Thornton, 1982; Hughes, 1987) and only an abbreviated discussion is presented here. The idea is to approximate the unknown variables appearing in the governing equations by a finite-dimensional set of basis functions with compact support (each basis function is non-zero in a small subdomain of the complete computational domain). This latter feature distinguishes the FEM from similar schemes, such as the Raleigh-Ritz method. The FEM also involves subdividing the computational domain into an assemblage of subdomains, the finite elements. This representation by a finite-dimensional basis is approximate, and results in some error, called a residual. In the general method of weighted residuals, this residual is required to be zero in a weighted-average sense by forming the so-called weak-form residual equation. The weak-form residual equation for the water balance is defined by

$$
R_{w, I}=\int_{\Omega} \Phi_{I}\left(\frac{\partial d_{w}}{\partial t}+\nabla \bullet F_{w}-Q_{w}\right) d \Omega,
$$

where $I=1, \ldots, N$, and $N$ is the number of weight functions, $\Phi_{I}$. In this equation, $\Omega$ denotes either the domain of a finite element, or the entire domain under consideration. Approximating variables by the same finite-dimensional basis set, which defines the GFEM, results in

$$
\begin{aligned}
R_{w, I}= & \int_{\Omega} \Phi_{I}\left(\sum_{J} \Phi_{J} \dot{d}_{w, J}\right) d \Omega-\int_{\Omega} \nabla \Phi_{I} \bullet F_{w} d \Omega \\
& -\int_{\Omega} \Phi_{I} Q_{w} d \Omega+\int_{\Gamma} \Phi_{I}\left(F_{w} \bullet n\right) d \Gamma
\end{aligned}
$$

where $\dot{d}_{w, J}$ is the time-rate of change of the bulk water density at nodal point $J$, and $\boldsymbol{n}$ is the outward pointing normal to the boundary surface of $\Omega$, denoted $\Gamma$. The flux divergence term has been integrated by parts, resulting in the boundary integral, which is useful for specifying a boundary flux to the domain. Although not explicitly denoted in the foregoing expression, it is understood that the variables are numerical approximations to the exact variables appearing in the original PDE, and that each is approximated by the same finite-dimensional basis set (see for example Eq. (37) below). A discrete nonlinear system of time-dependent ordinary differential equations is obtained by requiring each weak residual equation be zero, $R_{w, I}=0, I=1, \ldots, N$. The principal behind this weighted residual method is to require the vector of weight functions to be orthogonal to the water balance residual equation, thereby enforcing each weak residual equation to be zero in a weighted average sense. In a similar manner, the expression for the weak-form residual for the air balance appears as, 


$$
\begin{aligned}
R_{a, I}= & \int_{\Omega} \Phi_{I}\left(\sum_{J} \Phi_{J} \dot{d}_{a, J}\right) d \Omega-\int_{\Omega} \nabla \Phi_{I} \bullet F_{a} d \Omega \\
& -\int_{\Omega} \Phi_{I} Q_{a} d \Omega+\int_{\Gamma} \Phi_{I}\left(F_{a} \bullet n\right) d \Gamma
\end{aligned} .
$$

Calculation of the bulk densities, $d_{w}$ and $d_{a}$, will be discussed in the following section on time integration. The net component fluxes appearing in the mass balance equations are computed in terms of the phase mass fluxes and diffusion fluxes (c.f. Eq. (10)),

$$
\begin{aligned}
& F_{w}=\sum_{\beta} Y_{w \beta} \rho_{\beta} v_{\beta}+J_{w g} \\
& F_{a}=\sum_{\beta} Y_{a \beta} \rho_{\beta} v_{\beta}+J_{a g},
\end{aligned}
$$

where $\beta$ denotes the phase, liquid or gas. The finite element approximations to the phase mass fluxes are given by,

$$
\begin{gathered}
\rho_{l} v_{l}=-\frac{\rho_{l} k_{r l}}{\mu_{l}} k \cdot\left(\left(\sum_{J} \nabla \Phi_{J} P_{l, J}\right)+\rho_{l} g\right) \\
\rho_{g} v_{g}=-\frac{\rho_{g} k_{r g}}{\mu_{g}} k \cdot\left(\left(\sum_{J} \nabla \Phi_{J} P_{g, J}\right)+\rho_{g} g\right),
\end{gathered}
$$

for the liquid and gas phases, respectively. Gas phase binary diffusion fluxes are approximated by,

$$
J_{\alpha g}=-\rho_{g} D_{\alpha g}\left(\sum_{J} \nabla \Phi_{J} Y_{\alpha g, J}\right)
$$

where $\alpha$ denotes the chemical component, water or air in this case.

Applying the finite element approximations to the energy equation results in the weak form discrete energy balance,

$$
R_{e, I}=\int_{\Omega} \Phi_{I}\left(\sum_{J} \Phi_{J} \dot{e}_{J}\right) d \Omega-\int_{\Omega} \nabla \Phi_{I} \bullet q d \Omega-\int_{\Omega} \Phi_{I} Q_{e} d \Omega+\int_{\Gamma} \Phi_{I}(q \bullet n) d \Gamma .
$$

The net heat flux includes heat conduction, sensible and latent heat convection and heat transport due to binary gas diffusion, respectively,

$$
q=-\lambda_{T} \sum_{J} \nabla \Phi_{J} T_{J}+\sum_{\beta} \rho_{\beta} v_{\beta}\left(\sum_{J} \Phi_{J} h_{\beta, J}\right)+\sum_{\alpha} J_{\alpha g}\left(\sum_{J} \Phi_{J} h_{\alpha_{g}, J}\right),
$$


where $\beta$ denotes the phase, liquid or gas, and $\alpha$ the chemical component, water or air, $h_{\beta}$ are the phase enthalpies, and $h_{\alpha g}$ denotes the enthalpy of component $\alpha$ as it would exist pure at the same temperature and pressure of the gas phase. The discrete convective and diffusive fluxes are given by Eq. (32) through Eq. (34).

In the foregoing equations, the constitutive properties are also assumed to be represented with respect to the same basis as the bulk densities and pressure variables, for example,

$$
f(U)=\Phi^{T} f=\sum_{J} \Phi_{J} f\left(U_{J}\right),
$$

in which $f$ is replaced by $Q_{\alpha}, \rho_{\alpha}, k_{r \alpha}, \mu_{\alpha}, D_{\alpha g}$ and $\lambda_{T}$ in the foregoing equations, and $U$ denotes an unknown solution variable (primary variable).

To summarize, the GFEM discretization results in a set of time-dependent nonlinear ordinary differential equations,

$$
\begin{aligned}
{\left[\begin{array}{c}
R_{w, I} \\
R_{a, I} \\
R_{e, I}
\end{array}\right]=} & \int_{\Omega} \Phi_{I} \sum_{J} \Phi_{J}\left[\begin{array}{c}
\dot{d}_{w, J} \\
\dot{d}_{a, J} \\
\dot{e}_{J}
\end{array}\right] d \Omega-\int_{\Omega} \nabla \Phi_{I} \bullet\left[\begin{array}{c}
\boldsymbol{F}_{w} \\
\boldsymbol{F}_{a} \\
\boldsymbol{q}
\end{array}\right] d \Omega \\
& -\int_{\boldsymbol{\Omega}} \Phi_{I}\left[\begin{array}{c}
Q_{w} \\
Q_{a} \\
Q_{e}
\end{array}\right] d \Omega+\int_{\Gamma} \Phi_{I}\left[\begin{array}{l}
\boldsymbol{F}_{w} \bullet \boldsymbol{n} \\
\boldsymbol{F}_{a} \bullet \boldsymbol{n} \\
\boldsymbol{q} \bullet \boldsymbol{n}
\end{array}\right] d \Gamma,
\end{aligned}
$$

where the variables refer to the values at the current time step. In order to integrate this system in time, a time discretization method is needed for approximating the time derivatives of the bulk densities at the grid points.

\subsection{Time Integration}

\subsubsection{Predictor/corrector methods}

The time integration is achieved by using finite-difference approximations for time discretizations. In this work, we consider a variable-step, predictor-corrector method, first described by Gresho, et al., (1980). Two time integration methods are implemented. A first order scheme employs a forward Euler predictor with a backward Euler corrector. A second order scheme employs an Adams-Bashforth predictor with a trapezoid rule corrector. The predictors are used to obtain an initial estimate of variables at the next time step, $t_{n+1}$, thereby improving the initial estimate of the solution vector for use in a Newton iteration scheme applied to the nonlinear system of equations. An added benefit of these methods is that they provide a convenient way to implement a truncation-error-based method for timestep selection. In the second-order method, the predicted variables for step $n+1$ are given by the Adams-Bashforth predictor, 


$$
\boldsymbol{U}^{p, n+1}=\boldsymbol{U}^{n}+\frac{\Delta t_{n}}{2}\left(\left(2+\frac{\Delta t_{n}}{\Delta t_{n-1}}\right) \dot{U}^{n}-\frac{\Delta t_{n}}{\Delta t_{n-1}} \dot{\boldsymbol{U}}^{n-1}\right)
$$

where $\boldsymbol{U}$ denotes the vector of unknown solution variables at a grid point, and the $n$ superscript on $\boldsymbol{U}$ denotes the time level, the $p$ superscript denotes a predictor vector, and the time step sizes are defined according to $\Delta t_{n}=t_{n}-t_{n-1}$, etc. The first-order predictor is the standard forward Euler formula. The choice of solution variables requires special consideration and is discussed in the sequel. For present purposes it is sufficient to define the algorithm in terms of an as yet unspecified solution vector. The variable-step corrector equation is defined as,

$$
\dot{U}^{n+1}=\frac{\eta}{\Delta t_{n}}\left(U^{n+1}-U^{n}\right)-(\eta-1) \dot{U}^{n}
$$

where $\eta=1$ for the first-order backward Euler (BE) method, and $\eta=2$ for the secondorder trapezoid rule. The first-order method requires the solution vector from the previous time step, while the second-order method also requires the time-derivative of the solution vector from the previous time step. Time discretization in the FE equations is achieved by applying the variable-step corrector for approximating the time derivatives of the capacitance terms (time derivatives of bulk densities and energy). If we denote the vector of bulk densities and energy at a grid point by $D=\left(d_{w}, d_{a}, e\right)^{T}$, the grid-point time derivatives are approximated by

$$
\dot{D}_{j}=\sum_{i=1}^{N_{D O F}} \frac{\partial D_{j}}{\partial U_{i}} \dot{U}_{i}, \quad j=1, \ldots, N_{D O F}
$$

in terms of the "accelerations" ( $\dot{U})$ given by the corrector Eq. (40).

The predictor equations require that one or more vectors from previous time steps be available for their application in estimating variables at the next time step. That is, a startup scheme is needed. In this work, the first step uses the initial condition as predictor, the second step uses the FE predictor with $\mathrm{BE}$ corrector. At the third step, two previous time derivative vectors are available for use in either of the user-specified predictor/corrector pairs.

\subsubsection{Time step control}

The predictor/corrector scheme provides a method for estimating the local time truncation error, thereby providing a rational scheme for automatic time step control based on a userspecified truncation error tolerance. By using a norm-type analysis, the local time truncation error is estimated from the error norm between the predictor and corrector vectors. In this work the time truncation error norm is defined by 


$$
\left\|E_{t}\right\|=\left(\frac{1}{N_{u n k}} \sum_{i=1}^{N_{u n k}}\left(\frac{U_{i}^{p}-U_{i}}{\bar{U}_{i} \tau_{r}+\tau_{a}}\right)^{2}\right)^{\frac{1}{2}}, \quad \bar{U}_{i}=\frac{U_{i}^{p}+U_{i}}{2},
$$

where $N_{\text {unk }}$ is the global number of unknowns, $\tau_{r}$ and $\tau_{a}$ are relative and absolute userspecified error tolerances. The next time step size is specified so that the estimated time truncation error equals the user-specified error (Gresho et al., 1980), resulting in the formula

$$
\frac{\Delta t_{n+1}}{\Delta t_{n}}=\left(\frac{\varepsilon_{t} \beta}{\left\|E_{t}\right\|}\right)^{n}
$$

where $\varepsilon_{t}$ is a user-specified time truncation error tolerance, $\beta=2$ and $n=1 / 2$ for the first-order method, and $\beta=3\left(1+\Delta t_{n-1} / \Delta t_{n}\right)$ and $n=1 / 3$ for the second-order method.

\subsection{Primary Variables}

Substitution of Eq. (41) into Eq. (38) results in a discrete system of nonlinear equations whose solution describes nonisothermal flow of water and air through a porous material. There are three degrees of freedom per node point. Note that there are many more unknown nodal quantities than the three degrees of freedom per node. The various equations of state, thermodynamic functions and transport relations given in the preceding chapter must be applied to reduce the total number of unknowns per node to three.

Another not so obvious complication is that the state variables which uniquely describe the thermodynamic state of the system depend on which phases are present. The twophase, two-component system can exist in three possible states, two single-phase states (all-liquid or all-gas) and a two-phase state. Clearly, all three states could exist simultaneously in different parts of a computational domain. The system of nonlinear equations is to be solved for a set of primary variables, which by definition are a set of independent variables such that all other secondary variables can be computed from the primary variables. Unfortunately. common choices such as temperature and phase pressures, do not form a consistent set for all three possible states. For example, choosing the temperature and the liquid and gas; phase pressures as primary variables is fine for a two-phase state. However, the liquid phase pressure would be undefined in a co-existing all-gas state. In this latter state. a consistent set of primary variables could be derived by substituting a component mass fraction for the liquid pressure. Two techniques can be applied to solve this type of system. One is substitution of variables (Coats, 1978), wherein primary variables are chosen dynamically, depending on the state of the system at the grid point under consideration. The preceding discussion of primary variables is just such a case. If upon addition of sufficient heat such that all liquid is evaporated at a grid point, the algorithm must diagnose the change of state from two-phase to single phase gas and make the required substitution of primary variable consistent with the new thermodynamic state. Besides complicating the programming for such an algorithm, this scheme makes implementation of higher-order, variable-step time integration methods difficult and com- 
putationally expensive, requiring computing, storing, and communicating (for parallel algorithms) time derivative data for the accumulation (time) terms in the equations.

A second solution to the primary variables problem, and the scheme implemented in this work, is the use of a set of persistent variables, i.e., a set of primary variables valid for all possible states. The grid-point solution vector chosen is $U_{J}=\left(d_{w}, P_{a}, T\right)_{J}^{T}$, which allows the computation of all secondary variables for any of the three possible thermodynamic states.

\subsection{Nonlinear and Linear Solution Procedures}

\subsubsection{Inexact Newton iteration}

Having specified the primary variables, the task is to find the solution vector $U$ that minimizes the global residual vector, $\boldsymbol{R}=\left(\boldsymbol{R}_{w}, \boldsymbol{R}_{a}, \boldsymbol{R}_{e}\right)^{T}$, given by Eq. (38) once the time derivatives have been discretized. The discretized system of nonlinear equations can be solved for these variables by Newton's method. By Taylor series expansion of the system of nonlinear equations about the desired solution (i.e. the value of $U$ that minimizes the vector of residual equations), the system to be solved is found to be

$$
\boldsymbol{J}\left(\boldsymbol{U}^{q}\right) \delta U^{q+1}=-\boldsymbol{R}\left(\boldsymbol{U}^{q}\right),
$$

where $J$ is the Jacobian matrix,

$$
J_{i j}=\frac{\partial R_{i}}{\partial U_{j}}
$$

The solution vector is updated at each iteration $(q)$ according to

$$
U^{q+1}=U^{q}+\delta U^{q+1}
$$

until convergence is achieved; the stopping criteria is

$$
\frac{1}{N_{u n k}} \sum_{i=1}^{N_{u n k}} \frac{\left|\delta U_{i}\right|}{\left|U_{i}\right| \varepsilon_{r}+\varepsilon_{a}}<1,
$$

where $N_{u i k}$ is the global number of unknowns. The Jacobian can be computed efficiently via forward difference approximations, by exploiting the fact that most terms are sums of products of basis functions and grid variables. In this scheme, Eq. (45) is approximated by

$$
\frac{\partial R_{i}}{\partial U_{j}} \approx \frac{R_{i}\left(U+\delta U e_{j}\right)-R_{i}(U)}{\delta U},
$$


where $\boldsymbol{e}_{j}$ is a unit basis vector for the $j$-th unknown in the solution vector. This "inexact" Newton scheme (the term inexact in the present context refers to a numerical approximation of the Jacobian) is a convenient method of determining the Jacobian because any new transport parameter function or equation of state can be implemented without the need for the user to also program the gradient of the functions with respect to the solution vector variables. This is particularly helpful in the present class of problems where secondary variable calculations depend on which phases are present at a particular node point. However, special care must be taken in computing the forward differences to minimize finiteprecision errors (see for example, Gill et al. 1981, or Press et al., 1992). The error in the forward difference approximation is due to truncation and cancellation error. The former is proportional to the step size, while the latter varies inversely with the step size. The error is minimized by choosing the step size in the form $\delta U \approx|U| \varepsilon_{r}+\varepsilon_{a}$, with $\varepsilon_{r}$ roughly equal the square-root of the machine precision and $\varepsilon_{a}$ is a minimum step size. See Gill, et al., (1981) for a more complete discussion.

\subsubsection{Linear systems solvers}

The Newton iteration scheme generates a linear system of equations, viz. Eq. (44), to be solved for each update vector. The systems are solved using a parallel processing preconditioned Krylov solver library called Aztec (Hutchinson et al., 1995). The library includes several parallel iterative solution methods, including the conjugate gradient method for symmetric positive definite systems and a number of related methods for nonsymmetric systems, e.g. the generalized minimum residual method (GMRES) and transpose-free quasi-minimum residual method (TFQMR). The library includes several preconditioners

(e.g., Jacobi, least-squares polynomial, incomplete LU decomposition), which can be "mixed and matched" with the Krylov methods. An additional convenient feature is a scaling option, which is useful for the systems generated by the discretized multiphase equations which can yield poorly scaled linear equations. See Hutchinson et al., 1995 for additional information.

\subsection{Boundary Conditions}

Both Dirichlet and specified flux boundary conditions can be imposed on the discrete equations. Several combinations of Dirichlet and flux conditions are also allowable. The several types are discussed in the following.

\subsubsection{Dirichlet conditions}

Fixed values for some or all degrees-of-freedom at a particular boundary node can be specified directly into the residual equation for that unknown. For the Newton iteration, the residual equation in the Jacobian matrix corresponding to the bcundary node is replaced by an identity specifying the desired value. A general implementation of this method is to simply specify the residual equation for the boundary node as $R_{I}=u_{I}-g(t)$, where $u_{I}$ is the current value of the unknown and $g(t)$ is the Dirichlet value to be set. Clearly, the corresponding Jacobian entry is unity on the diagonal and zero elsewhere. Each matrix solution and update will enforce the desired Dirichlet value. This technique 
can obviously be applied to the discretized system for both constant and time-varying Dirichlet values.

\subsubsection{Flux conditions}

Specified surface fluxes are the "natural boundary conditions" for the FE formulation shown earlier. Surface fluxes are conveniently applied by specifying values for the boundary flux terms appearing in Eq. (38). In general the fluxes are time dependent, and can depend on the solution vector at the considered node. Note that these terms specify the net component flux, in the case of the component mass balance equations, or the total heat flux in the case of the energy equation. For example, a surface energy flux can be applied by specifying $q \bullet n$ in the term

$$
\int_{\Gamma} \Phi_{1} q \bullet n d \Gamma
$$

appearing in Eq. (38). However, in view of Eq. (36), this value embodies both convection and diffusion of heat. If the surface is impermeable, the diffusive heat flux is specified. If there is mass flow at the surface, the heat flux value represents both convective and diffusive transport of energy. The fluxes can be specified in terms of transfer coefficients in the form,

$$
q \bullet n=h\left(T-T_{r e f}\right)
$$

for the case of energy transport, and in analogous forms for the mass balance equations. If the parameters are dependent on the solution variables in a general way, a user-supplied function can be included to allow such an application. For example, wells are often approximated by setting fluxes which are proportional to the difference between the boundary pressure in the computation and a wellbore pressure. The coefficient for the flux may also depend on the boundary pressures, temperature, and fluid saturations, see Aziz and Settari (1979).

Combinations of surface fluxes and Dirichlet conditions can also be applied, so long as they form a mathematically consistent set of boundary conditions. An example of an improper specification would be to specify mass inflow at a surface with zero energy flux. The latter condition would imply zero temperature for the inflowing fluids.

\subsection{Parallel Implementation}

The foregoing numerical algorithm is implemented for distributed memory parallel computers, or networked systems, via domain decomposition and message-passing techniques (e.g., Foster, 1995). In this work we used the MPSalsa code (Shadid et al., 1996) as a platform, including the parallel-processing implementation. However, there are several properties of the current problem which necessitated some special developments. A special data structure was necessary for the porous medium calculations. In our formulation it is necessary to have a unique material type specified on a node-point basis. This requirement 


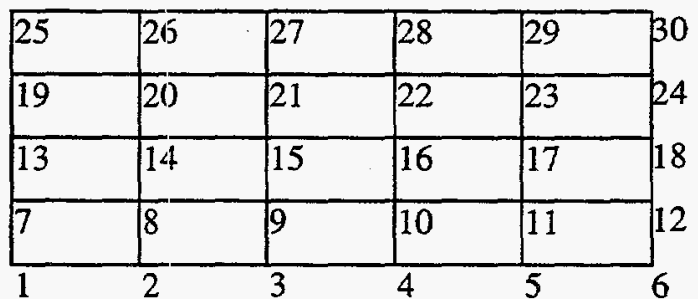

(a)

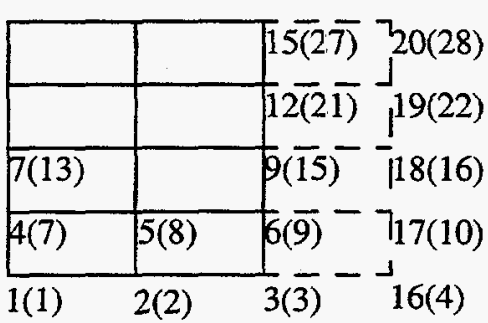

(b) Subdomain for processor $\mathrm{PO}$

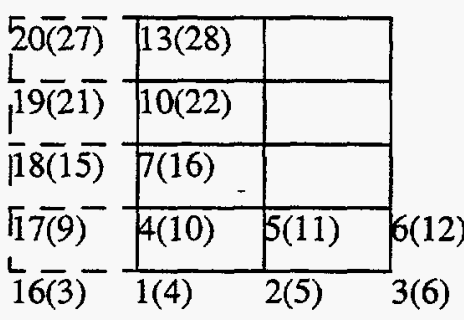

(c)Subdomain for processor P1

Figure 1 Schematic of a domain decomposition in which the global mesh (a) is decomposed into two subdomains, (b) and (c). The dashed elements denote the "ghost elements" on each partition. The global mesh displays the global node numbering scheme, whereas the subdomains display the processor-level node numbering, with the corresponding global node numbers displayed in parenthesis.

can be attributed to the capillary pressure vs. saturation constitutive model, which is nonunique at a material boundary, and our choice of primary variables. Our solution to accommodate this non-FEM data structure was to build a processor node-point-to-material mapping. To ensure a consistent mapping across processors, a inter-processor communication is required to update the correct mapping for the "ghost nodes" on each processor.

Domain decomposition, as used in the current context, refers to the process of breaking the geometrical computational domain into an assemblage of subdomains for the purpose of assigning the computational work for each subdomain to one unique processor. In a sense, this procedure is similar in spirit to the finite element method in which the domain is represented by an assemblage of finite elements, or subdomains of the full domain. The domain decomposition itself is performed with a modified version of the Chaco (Hendrickson and Leland, 1993) graph partitioning code. The task for Chaco is to decompose the global node set graph into a user-specified number of partitions (subgraphs) in such a way as to minimize the edge interactions between partitions.

A simplified schematic of a domain decomposition is shown in Figure 1 for a structured grid (the algorithm is implemented for general unstructured grids). The global domain is decomposed into two subdomains, wherein the set of nodes assigned to each processor are numbered sequentially, starting with the set of nodes that "belongs" to the processor, and with the ghost or external nodes numbered last. In the figure, the global node numbers corresponding to the processor-level node numbers on the mesh partitions (Figs. 1b and 1c) are shown in parenthesis. The dashed elements are the so-called "ghost elements" and the associated node points are referred to as "ghost nodes" or external nodes. Each processor 
is assigned the nodes corresponding to the un-dashed elements in Figure 1. However, on each processor the data for the external nodes is necessary to complete the processing for the border nodes, i.e. the surface nodes on each subdomain. Clearly, interprocessor communication is required for exchanging information associated with the ghost nodes, and those "border" nodes associated with the current processor.

The implementation of this communication requires for each processor a list of other processors with which data must be shared, and, for each of these processors, a list of nodes with which data is to be exchanged. For those familiar with finite element processing, these lists are reminiscent of the connectivity array (albeit a multi-level connectivity) relating which nodes in a mesh define a particular finite element. The interprocessor communication is set up to take advantage of the native Intel Paragon communication structures, enabling efficient communication on the Intel Paragon, and is also set up to use the standard message-passing library definition MPI (Gropp, et al., 1995), thus making the algorithm highly portable. The domain-decomposition itself is done in a pre-processing step, creating a file containing the decomposition information necessary for interprocessor communication.

The decomposition in Figure 1 suggests some features of the parallel performance associated with interprocessor communication. The amount of communication for a particular decomposition is proportional to the ratio of the number density of surface nodes to internal plus surface nodes in the partition. The relative amount of communication is analogous to the rate of heat loss of a volume, which is proportional to the surface area to volume ratio. For a user faced with solving a problem on a particular mesh, there will be a more-orless optimal number of processors (partitions) which will minimize run-time. Too few processors will render communication costs negligible but will over-load (assuming more processors are available) the work on each processor. With too many processors, communication costs will overwhelm CPU processing. With ever-increasing demands on resolution, the latter condition is less likely than the former.

\section{Numerical Results}

The results of verification studies and applications of the code are described here. The verification studies demonstrate the correct implementation of the numerical algorithms. Several illustrative applications are also included to demonstrate the parallel processing capabilities.

\subsection{Benchmark Problems}

\subsubsection{One-dimensional infiltration into a homogeneous porous material}

A typical test problem for unsaturated flow is that of one-dimensional infiltration of moisture into a homogeneous porous material. The version of the infiltration problem presented here was previously used in evaluating the solution approach in $\mathrm{JACQ}^{3} \mathrm{D}^{3}$, a computer program for solving nonlinear heat conduction problems. As part of the evaluation, results from JACQ3D were compared with those from a one-dimensional code, based on the 
method-of-lines (MOL), which solves isothermal unsaturated flow in fractured and unfractured porous media (Martinez, 1988). In this test problem, the initial saturation is assumed uniform in the material and moisture is introduced at the top of the column by specifying a saturated condition.

The material models specified for the relative permeability and capillary pressure follow the so-called van Genuchten function (van Genuchten, 1978), and method of Mualem (Mualem, 1976)

$$
k_{r}(\bar{S})=\sqrt{\bar{S}}\left[1-\left(1-\bar{S}^{\frac{1}{\lambda}}\right)^{\lambda}\right]^{2}
$$

and

$$
P_{c}(\overline{\bar{S}})=\frac{\rho_{l} g}{\alpha}\left[\bar{S}^{-\frac{1}{\lambda}}-1\right]^{\frac{1}{\beta}}
$$

with $\lambda=1-1 / \beta . g$ is the gravitational acceleration and $\rho_{l}$ is the liquid density. The scaled saturation, $\bar{S}$, is a function of the liquid saturation, $S$, and the material residual saturation, $S_{r}$

$$
\bar{S}=\frac{S-S_{r}}{1-S_{r}}
$$

The two parameters, $\alpha$ and $\beta$, influence the degree of nonlinearity in the problem. The former provides a pressure scale for capillary forces, while the latter is indicative of poresize distribution. Material properties and model parameters specified in this problem are shown in Table 1. The saturated conductivity listed in Table 1 is a term preferred by hydrologists and is defined as $K_{s}=\left(\rho_{l} g k\right) / \mu_{l}$. These properties are similar to a sand-like porous material. The computational mesh used for both the MOL code and the present code consisted of 20 elements, each one-half meter in length, for a total column length of 10 meters $(0 \leq z \leq 10 \mathrm{~m})$.

Figure 2 shows moisture content profiles at various times for Dirichlet boundary conditions, with $50 \%$ initial saturation. This value of initial saturation results in about two orders of magnitude variation in conductivity over the computational mesh. Also shown is the solution obtained the MOL code. There is good agreement between results. The MPSalsa solution required about 14 seconds on a SPARCstation 20/150 for 121 time steps.

3. J. H. Biffle, JACQ3D - A three-dinnensional finite element computer program for nonlinear heat conduction problems with the conjugate gradient method, unpublished SNL Tech. Rept.. 
Table 1: Hydrologic Properties

\begin{tabular}{|l|c|c|}
\hline Parameter & Value & Units \\
\hline \hline$\alpha$ & 0.10 & $\mathrm{~m}^{-1}$ \\
\hline$\beta$ & 2.0 & - \\
\hline$S_{r}$ & 0.1 & - \\
\hline$\varphi$ & 0.43 & - \\
\hline$K_{s}$ & $8.2 \times 10^{-5}$ & $\mathrm{~m} / \mathrm{s}$ \\
\hline
\end{tabular}

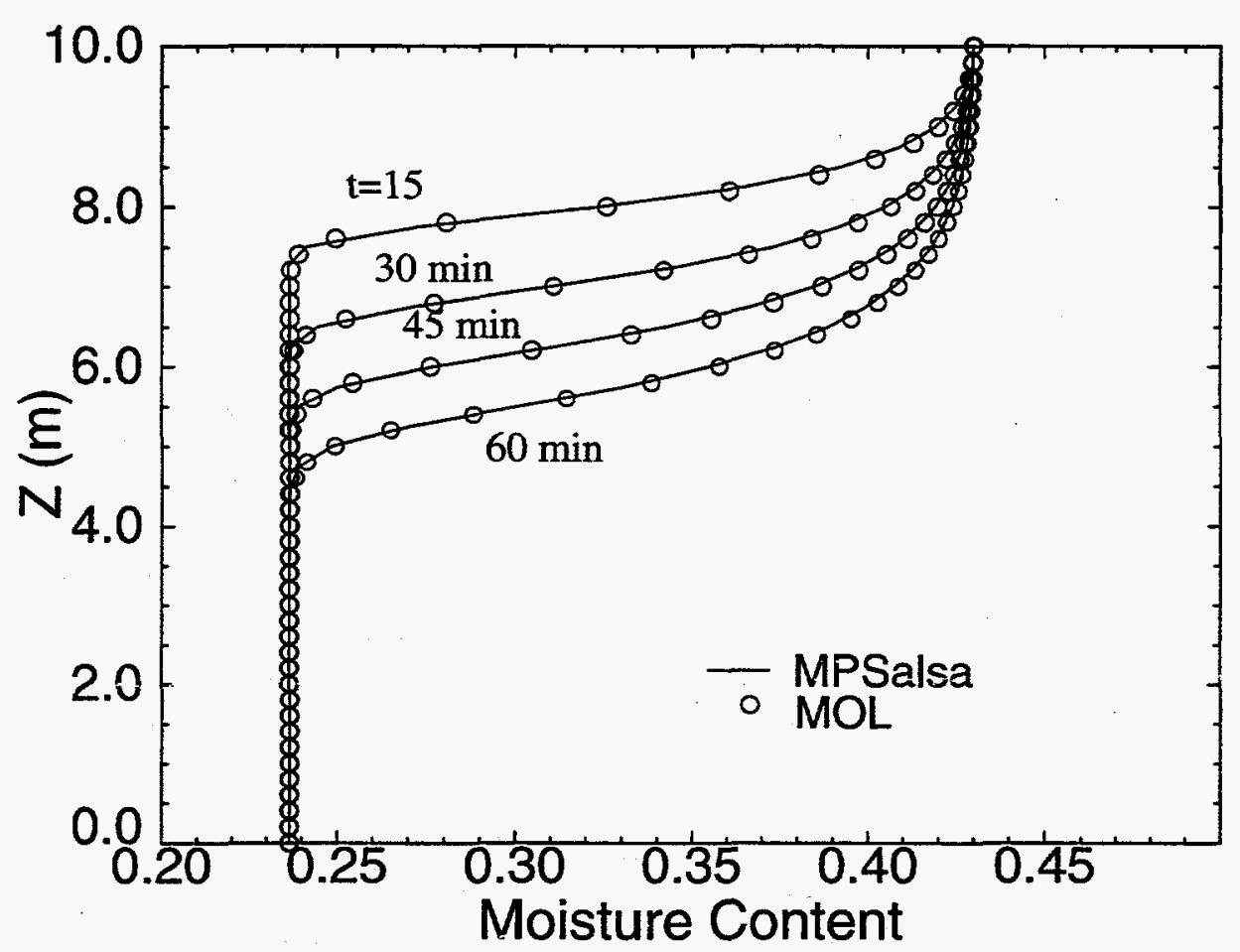

Figure 2 Moisture profiles of infiltration into a homogeneous porous material.

\subsubsection{One-dimensional infiltration into a layered medium}

This example involves one-dimensional steady infiltration into a layered porous medium. This problem is a particular case (case 2) of the Yucca Mountain project COVE 2A benchmarking study, see Hopkins (1990) for more details. The problem is driven by a $0.1 \mathrm{~mm} / \mathrm{yr}$ infiltration rate at the top of a $530 \mathrm{~m}$ column composed of the 4 major hydrostratigraphic units of Yucca Mountain, as were understood at the time of the study. In case 2, the material was considered to be unfractured. The material models specified for the relative permeability and capillary pressure follow the van Genuchten functions given in the previous section. 
Table 2: Hydrogeologic data for layered medium (COVE 2A, case 2).

\begin{tabular}{|l|l|l|l|l|l|l|}
\hline Geologic unit & $\begin{array}{c}\text { Elevation } \\
\text { interval (m) }\end{array}$ & $\phi$ & $k\left(\mathrm{~m}^{2}\right)$ & $\alpha\left(\mathrm{m}^{-1}\right)$ & $\beta$ & $S_{r}$ \\
\hline \hline Calico Hills & $0-130$ & 0.46 & $2.7 \times 10^{-14}$ & 0.016 & 3.872 & 0.041 \\
\hline $\begin{array}{l}\text { Topopah } \\
\text { Springs }\end{array}$ & $130-465$ & 0.11 & $1.9 \times 10^{-18}$ & 0.00567 & 1.798 & 0.01 \\
\hline Paint Brush & $465-505$ & 0.40 & $3.9 \times 10^{-14}$ & 0.015 & 6.872 & 0.10 \\
\hline Tiva Canyon & $505-530$ & 0.10 & $9.7 \times 10^{-19}$ & 0.00821 & 1.558 & 0.002 \\
\hline
\end{tabular}

Table 2 lists the relevant material data. Figure 3 compares profiles of liquid saturation as a function of elevation above the water table as obtained with the code LLUVIA (Hopkins and Eaton, 1990), used in the COVE 2A study, and the present code. The abrupt breaks in the profile mark the interfaces between the geologic units, and indicate significantly different hydrologic properties for each layer. Some of the discrepancies in the figure can be attributed to different mesh representations of the units. The exact location of interfaces is different by a half mesh spacing (about 5 meters) between the grids, resulting in slightly different layer thicknesses. Otherwise, the comparison is good.

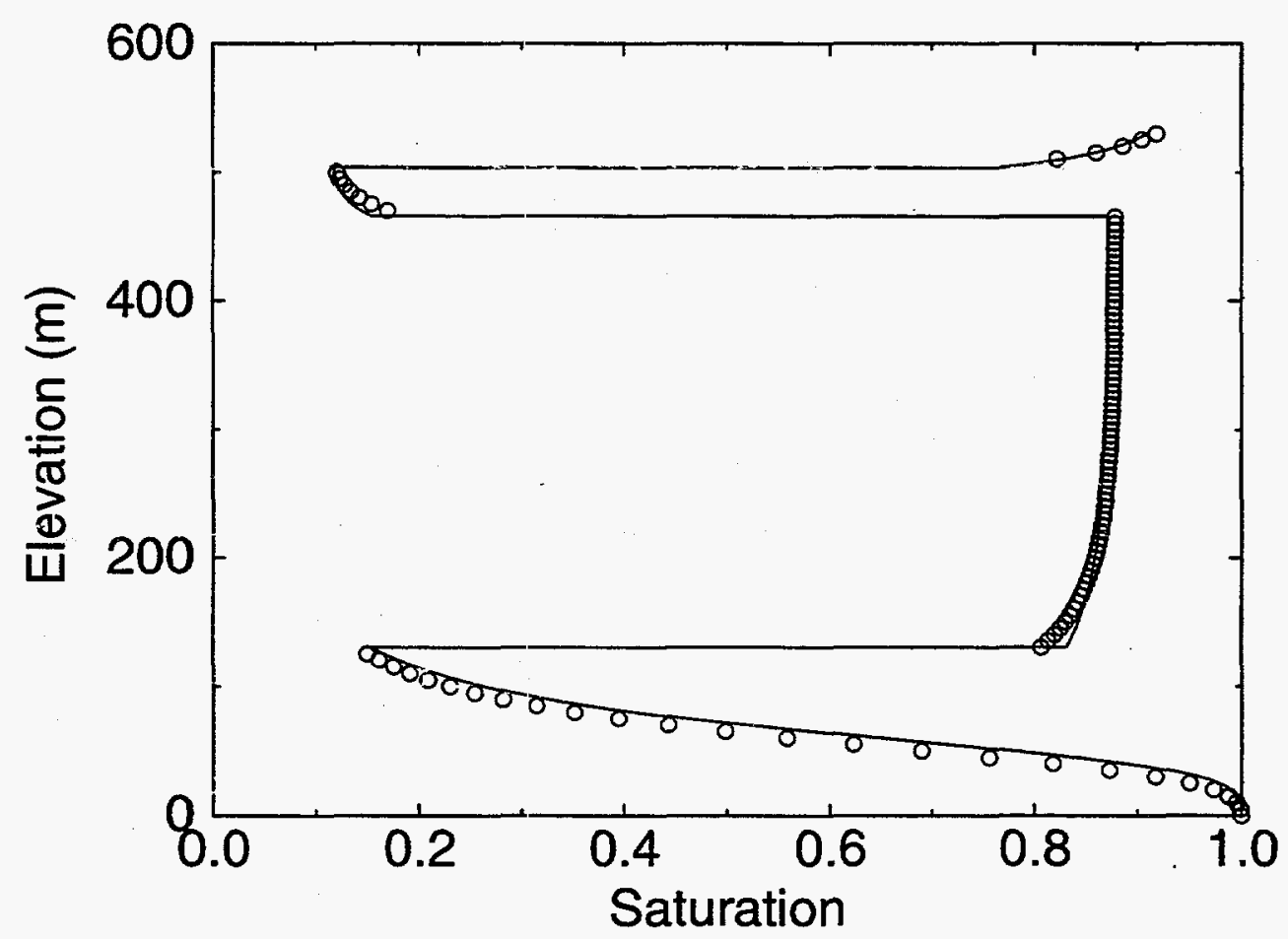

Figure 3 Comparison of Cove 2A, case 2 simulation between LLUVIA (solid line) and present results (symbols). 


\subsubsection{One-dimensional heat injection into a homogeneous porous material}

The steady heat pipe problem discussed by Udell and Fitch (1985) is the basis for the set of transient, two-phase flow test problems described here. These problems involve the injection of heat into a one-dimensional horizontal column of porous material in which the void volume is filled with air and water (liquid and vapor). These problems were solved previously (Martinez, 1995a) using the one-dimensional, method-of-lines code (MOL) described in Section 5.1.1, and the finite element computer program FEHMN (Zyvoloski et al., 1993).

The material properties specified for these problems are the same as in the steady heat pipe problems posed by Udell and Fitch (1985), and are described here. The capillary pressuresaturation relation is given by

$$
P_{c}=\sigma \sqrt{\frac{\phi}{k}}\left(1.417(1-s)-2.12(1-s)^{2}+1.263(1-s)^{3}\right)
$$

and the relative permeabilities are defined by

$$
k_{r g}=(1-s)^{3} \quad k_{r l}=s^{3},
$$

where $\sigma\left(=0.05878 \mathrm{~N} / \mathrm{m}^{2}\right)$ denotes surface tension and

$$
s=\frac{S_{l}-S_{l r}}{1-S_{l r}} .
$$

The material has $40 \%$ porosity $(\phi)$ and 1 Darcy permeability $\left(k=10^{-12} \mathrm{~m}^{2}\right)$. In addition, the effective thermal conductivity was specified as

$$
\lambda=\lambda_{0}+\sqrt{S_{l}}\left(\lambda_{1}-\lambda_{0}\right)
$$

with $\lambda_{0}=0.582 \mathrm{~W} / \mathrm{m}-\mathrm{K}$ and $\lambda_{1}=1.13 \mathrm{~W} / \mathrm{m}-\mathrm{K}$.

The first example problem involves the injection of heat into a one-dimensional horizontal column of porous material $2 \mathrm{~m}$ in length $(L)$. The void volume is filled with air and water (liquid and vapor). The end $(x=L)$ of the column opposite the heat injection is maintained at initial conditions, $T=70^{\circ} \mathrm{C}, P_{g}=1 \mathrm{~atm}(.10133 \mathrm{MPa})$, and $S_{l}=\theta_{l} / \phi=0.5$. A heat flux of $20 \mathrm{~W} / \mathrm{m}^{2}$ is applied at $x=0$; this boundary is also closed to flow of air and water. This value of heat flux is low enough that heat transport is mostly by conduction, however, vapor pressure gradients also contribute to mass and energy transport. A constant effective thermal conductivity of $\lambda=0.86 \mathrm{~W} / \mathrm{m}-\mathrm{K}$ was used in this problem. The $2 \mathrm{~m}$ column was discretized into 100 evenly spaced elements.

Figures 4 and 5 show the evolution of temperature in the column over a 30 day simulation period. Also shown are the results of the simulation of the problem using the finite element 
multiphase code FEHMN. For FEHMN, a two-dimensional 100-element mesh was specified for discretizing the computation region $(1 \times 2 \mathrm{~m})$, resulting in 202 node points at which the solution is calculated. The FEHMN calculations did not include binary diffusion in the gas phase; the simulation with the MOL code was performed with and without diffusion, as was the simulation with MPSalsa. The solutions without diffusion using FEHMN and the MOL compare well with those of MPSalsa (Figure 4). Comparing the results to those of Figure 5 also illustrates the non-negligible influence of binary diffusion in the gas phase for a problem driven by even a moderate heat flux. The hotter temperatures increase the vapor pressure and so provide a concentration gradient which drives diffusion of water vapor to the cold end of the column. There is a corresponding decrease of liquid water content (i.e., drying) near the heat source (not shown).

The second test problem is similar to the one just described, except that it involves a higher rate of heat injection. For purposes of comparison, the transient evolution to the steady solution was simulated and results compared with the MOL code. This problem exercises features of evaporation/condensation and vapor and liquid flows in the code. The material properties are the same as described in the preceding example. Binary diffusion in the gas phase is allowed, and the variable effective thermal conductivity of Eq. 54 is used. Once again, a $2 \mathrm{~m}$ horizontal column filled with water and air is considered, and the same discretizations as discussed above were used. Also, the same initial conditions were specified $\left(T=70^{\circ} \mathrm{C}, P_{g}=1 \mathrm{~atm}\right.$, and $\left.S_{l}=0.5\right)$, but the boundary conditions differ. To initiate the transport, the left end $(x=0)$ is abruptly saturated with liquid $\left(S_{l}=1\right)$, while the temperature and pressure are maintained at $70^{\circ} \mathrm{C}$ and $0.10133 \mathrm{MPa}$, respectively. A $100 \mathrm{~W} / \mathrm{m}^{2}$ heat flux is applied at $x=L$, which is also closed to flow of air and water.

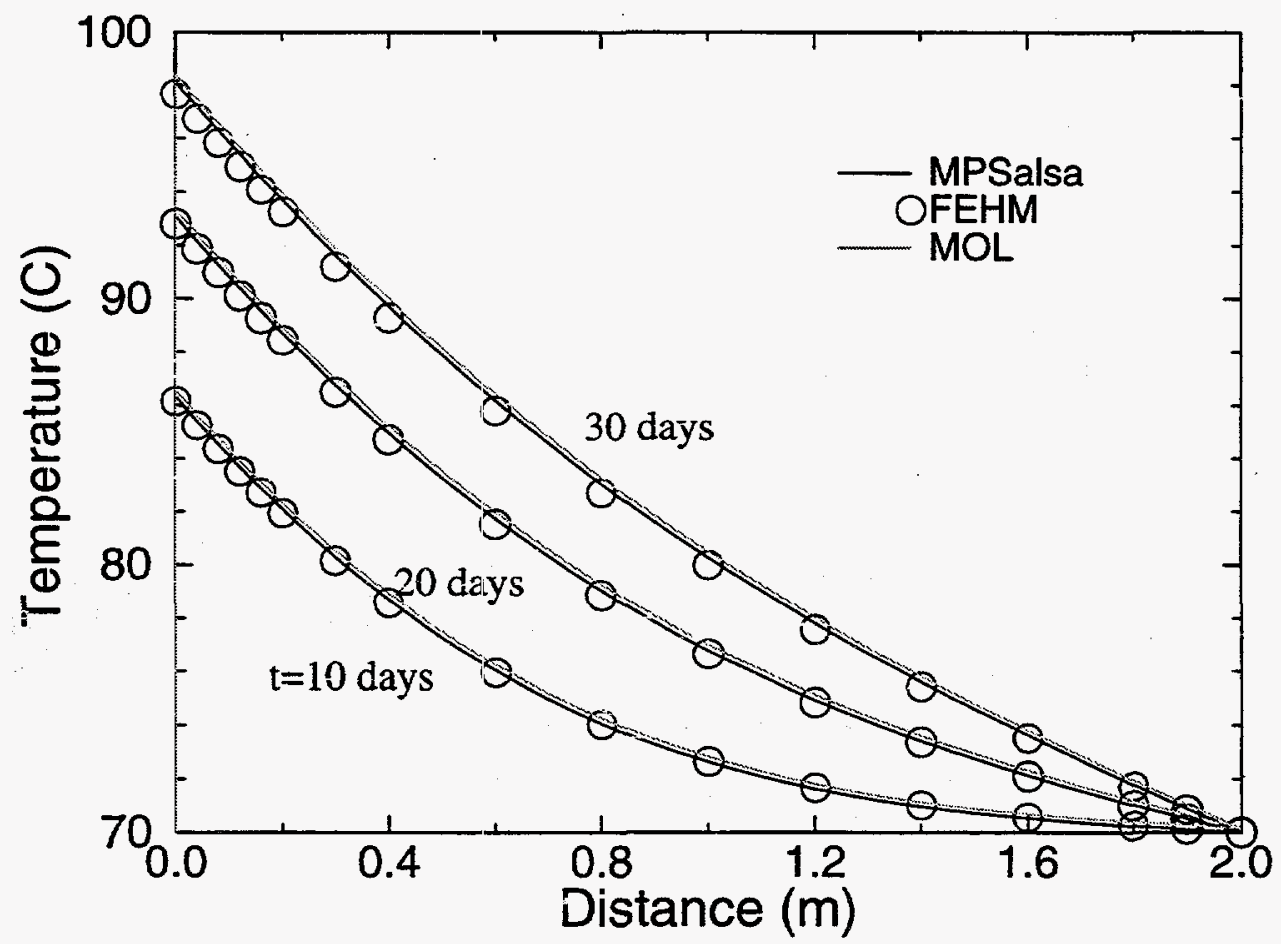

Figure 4 Temperature profiles due to a $20 \mathrm{~W} / \mathrm{m}^{2}$ heat flux (no binary diffusion). 


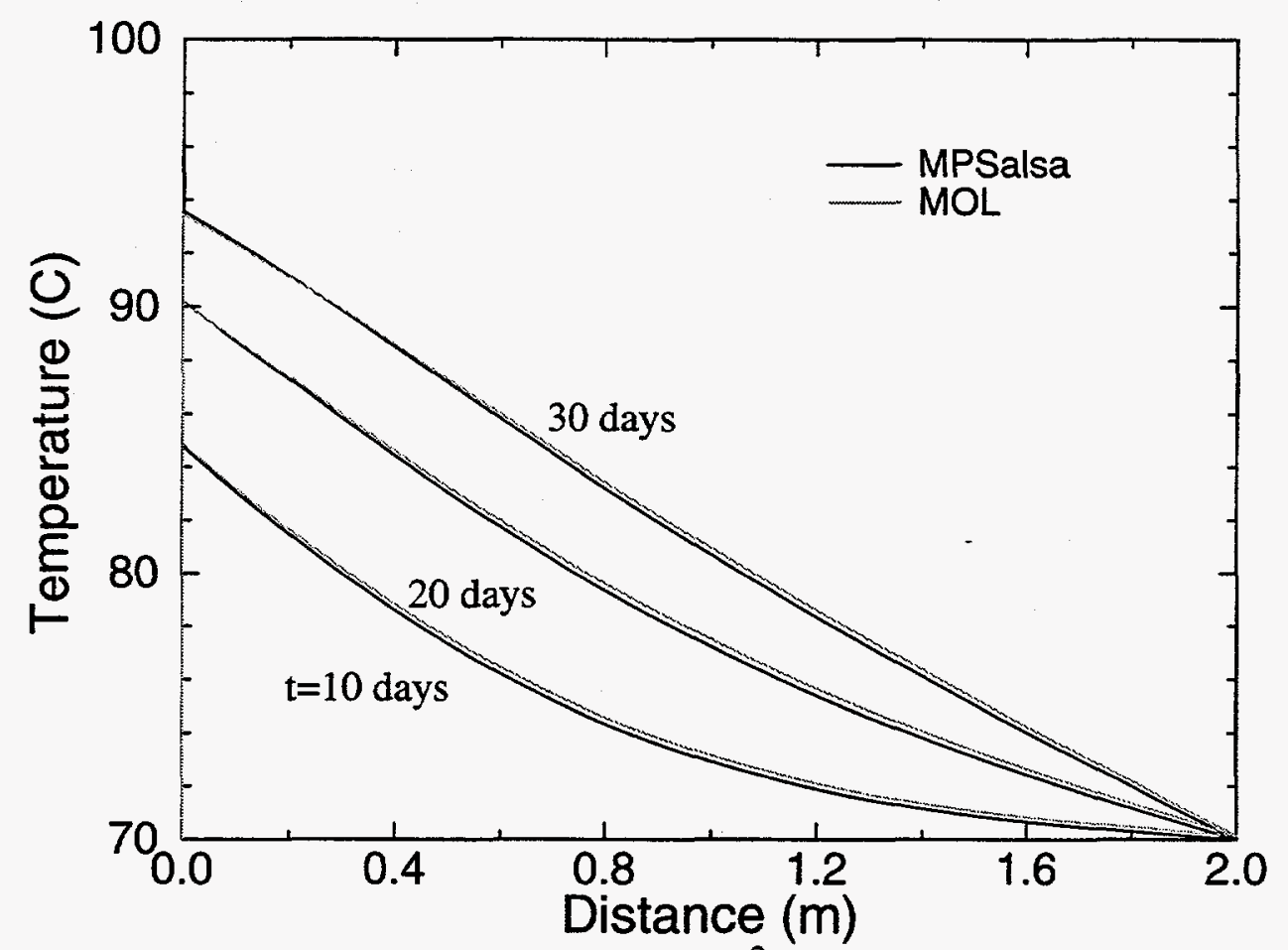

Figure 5 Temperature profiles due to a $20 \mathrm{~W} / \mathrm{m}^{2}$ heat flux (binary diffusion).

Figures 6 and 7 compare the transient evolution of liquid saturation and temperature as given by the MOL and MPSalsa codes for up to 50 days of simulation, by which time a steady solution is attained. It is noted that, for a two-meter column, neither the MPSalsa nor the MOL steady solutions (50 days) indicate complete dry-out of the porous material.

A more detailed description of the MOL and FEHMN calculations is presented in Martinez, (1995).

\subsubsection{Three-dimensional flow in unsaturated heterogeneous porous media}

The test problem considered here is one used in earlier benchmarking efforts in both 2-and 3-D domains. The geometry for the 2-D problem is shown in Figure 8. The geometry for the 3-D problem was obtained by simply extending the $x-z$ plane shown a distance of 2 meters in the 1 -direction. The four distinct material regions indicated in Figure 8 were retained along the $y$-direction. The material properties are given in Table 2. The 3-D mesh used in this study, which includes 18,900 eight-node brick elements and 21,758 nodes, is shown in Figure 9, and measures $8.5 \mathrm{~m}$ along $x, 6.5 \mathrm{~m}$ along $z$ and $2 \mathrm{~m}$ in the $y$-direction. The problem is 3D because of the applied boundary conditions. The boundary conditions are no-flow everywhere, except for a source area on the upper surface of the domain. The source patch is defined on $z=6.5 \mathrm{~m}$ over $0 \leq x \leq 2.25 \mathrm{~m}$ and $0 \leq y \leq 1 \mathrm{~m}$. An infiltration rate of $2 \mathrm{~cm} /$ day is specified over the patch. The problem was simulated for 30 days of infiltration. Initially, the porous medium is relatively dry with a uniform initial value of pressure head, $\psi=-7.34$. This problem was run both as a linear problem (constant 


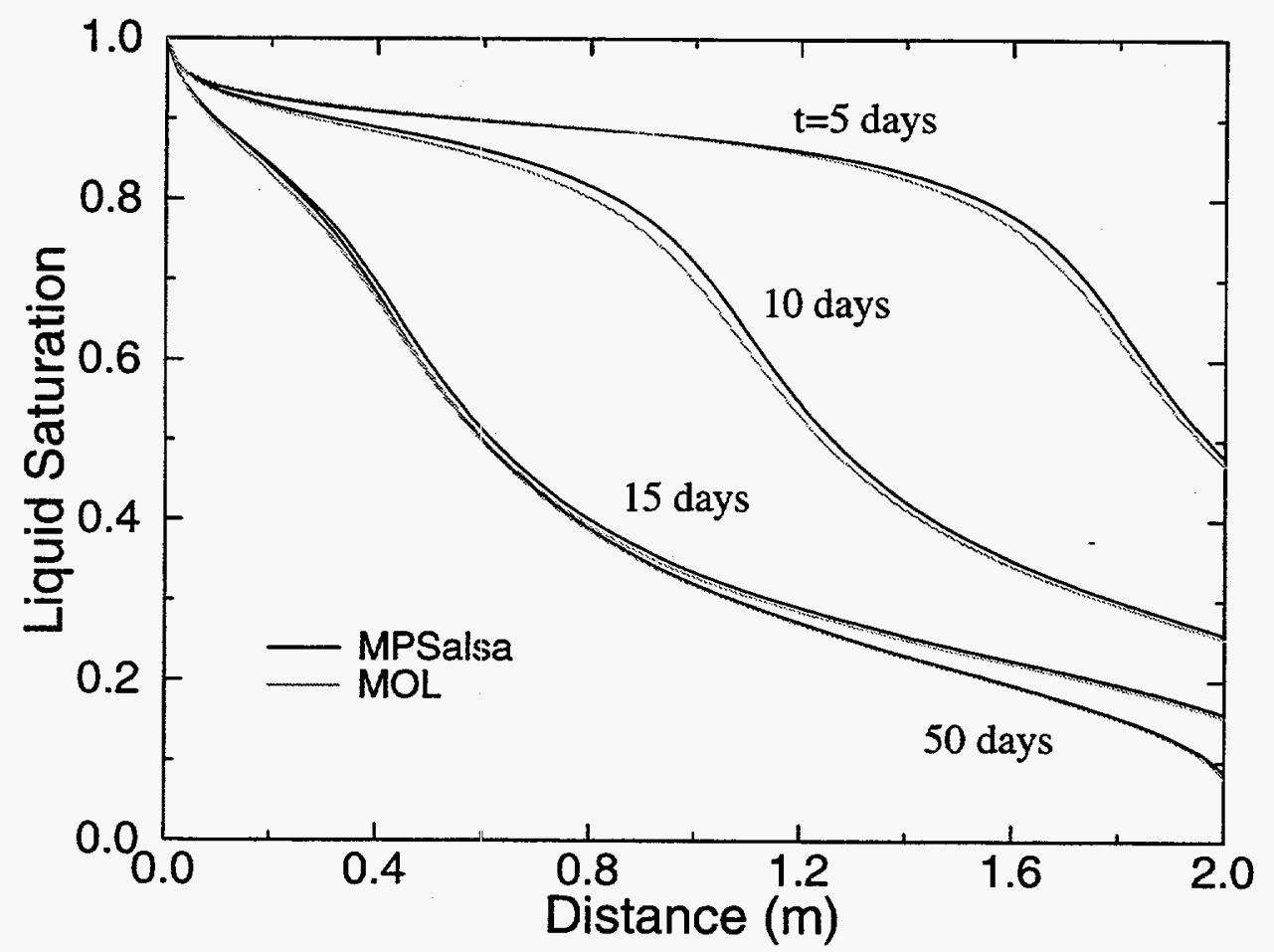

Figure 6 Saturation profiles due to a $100 \mathrm{~W} / \mathrm{m}^{2}$ heat flux (binary diffusion).

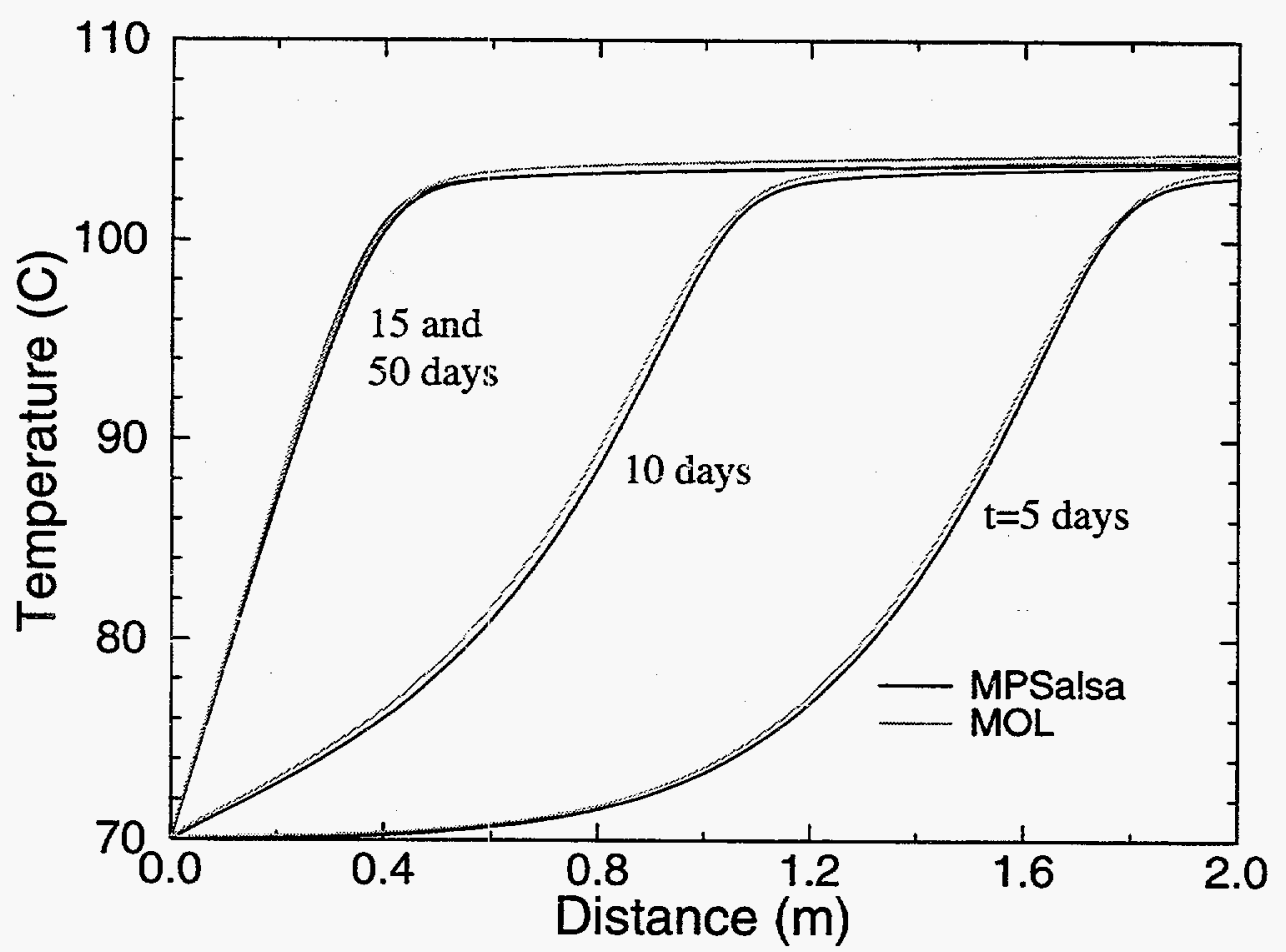

Figure 7 Temperature profiles due to a $100 \mathrm{~W} / \mathrm{m}^{2}$ heat flux (binary diffusion). 
Table 3: Hydraulic Properties

\begin{tabular}{|c|c|c|c|c|c|}
\hline $\begin{array}{c}\text { Material/ } \\
\text { Zone No. }\end{array}$ & $\begin{array}{c}K_{s} \\
\text { (m/day) }\end{array}$ & $\phi$ & $\theta_{r}$ & $\begin{array}{c}\alpha \\
\mathrm{m}^{-1}\end{array}$ & $\beta$ \\
\hline \hline 1 & 7.909 & 0.368 & 0.1020 & 3.34 & 1.982 \\
\hline 2 & 4.699 & 0.351 & 0.0985 & 3.63 & 1.632 \\
\hline 3 & 4.150 & 0.325 & 0.0859 & 3.45 & 1.573 \\
\hline 4 & 41.50 & 0.325 & 0.0859 & -3.45 & 1.573 \\
\hline
\end{tabular}

hydraulic conductivity, $K_{s}$ ) and a nonlinear problem (hydraulic conductivity a function of pressure head). A measure of the degree of nonlinearity in the solutions is the variation in conductivity over the solution domain. For the nonlinear case, simulations indicate a 4 to 5 order-of-magnitude variation in conductivity in material 1 , the region containing the source patch.

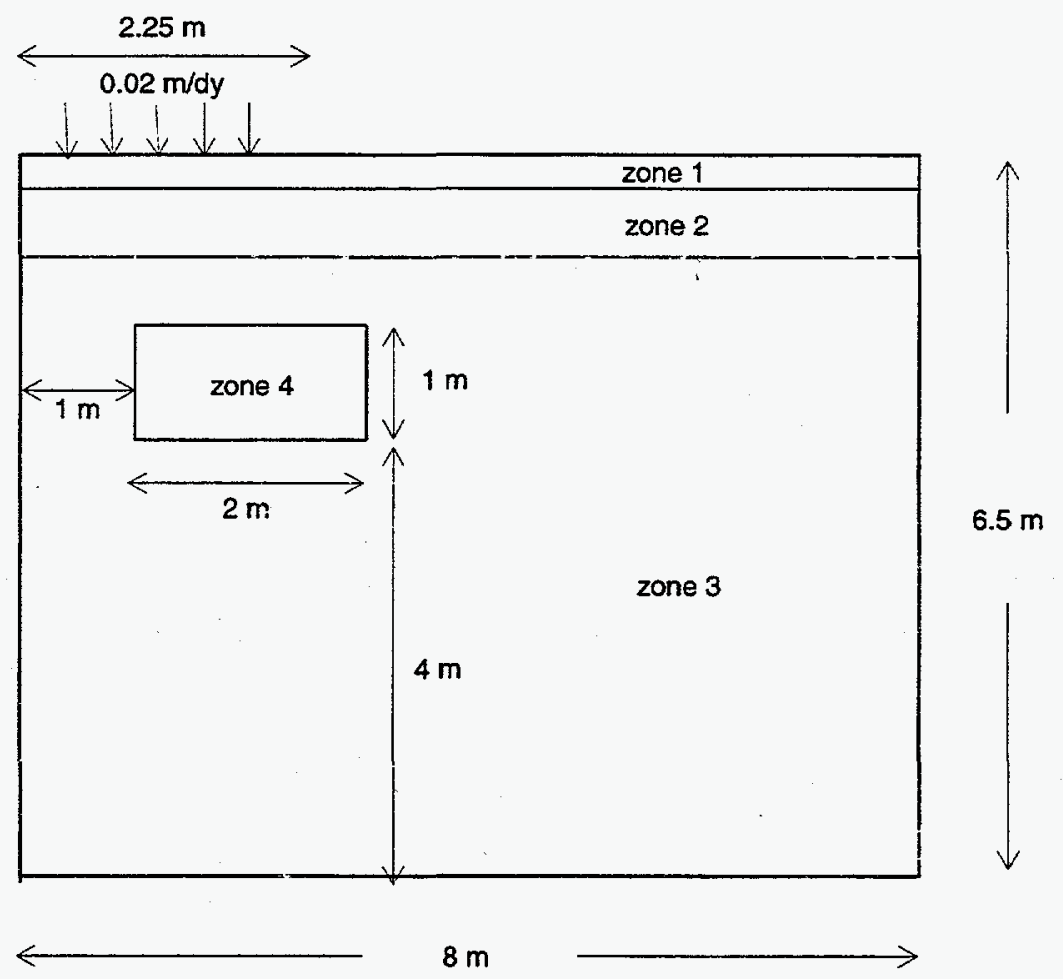

Figure 8 Schematic of two-dimensional problem. 


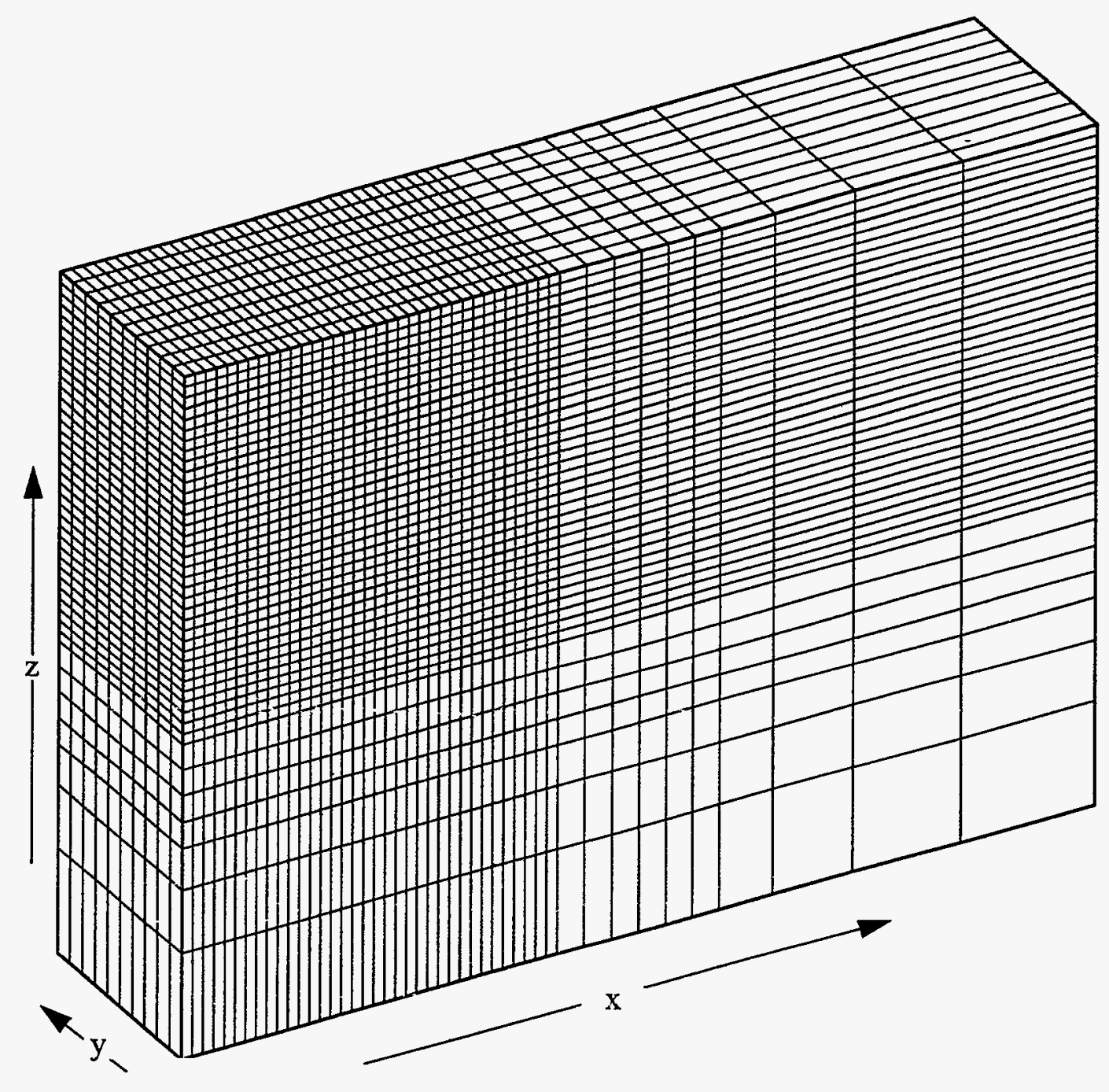

Figure 9 Finite element mesh for 3D problem (18,900 brick elements). 
Contours of saturation, $S_{l}$, at various times during the simulation of the nonlinear problem are shown in Figure 10 for illustrative purposes. The results show the general downward invasion of moisture introduced at the source into the initially dry material. Details of the infiltration can be explained by considering the contrast in material properties, as given in Table 2. Material 4, which is the rectangular inclusion, has the same material properties as the enclosing material (material 3), except the saturated conductivity is ten times larger. At 10 days the effect of the large conductivity is evident as Figure 10 shows the faster invasion of the moisture through the inclusion. By the same reasoning, at 20 days the moisture is seen to be propagating along the $x$-direction in the inclusion, since the moisture has infiltrated from the upper left corner (in the $y=0$ plane) and rapidly dropped down to the lower left edge with the help of gravity and finally diffuses laterally inside the inclusion. At 30 days, the lower left corner retains a "perched"' (local maximum in saturation) pocket because of the high-to-low conductivity contrast between materials 4 and 3 . The region in material 3 directly above the inclusion is drained (i.e. a local minimum in saturation) by the high conductivity inclusion. Figure 11 compares saturation contours at 30 days computed with Coyote II (top figure, D. K. Gartling and R. E Hogan, 1994), and the present code (bottom figure). Since Richards equation can be cast as a nonlinear heat conduction problem, Coyote II (a nonlinear heat conduction code) can be used to model isothermal unsaturated flow by defining hydrologic properties as pseudo thermal properties.

\subsection{Illustrative Applications}

Having verified the implementation of nonisothermal two-phase flow in the code, we now turn to some illustrative examples intended to demonstrate some of the capabilities of this simulator.

\subsubsection{Pre-emplacement infiltration at Yucca Mountain}

Because of its unique capabilities, the code was applied to a high-resolution, fractured flow study in cross-sections of Yucca Mountain (YM) in southwestern Nevada, a location being considered for the nation's first high-level nuclear waste repository. This application was part of a larger multi-scale study to evaluate scaling issues at YM (Altman, et al. 1996). The main objective of the present study was to investigate "lateral flow" at YM as it affects the percolation flux at the proposed repository horizon. Flow simulations including a complete geostratigraphical description on the mountain-scale (as is presently understood) had not been previously possible due to computer resolution limitations.

The east-west cross section considered in the study is shown in Figure 12, in which 19 different hydrogeologic units were modeled. Each of the major geologic units indicated in the figure is composed of several sub-units. The particular area of interest for the study is the Paint Brush unit, which is labelled in the figure, and which is composed of several subunits, some of which pinchout along the lateral direction. The numerical grid for this cross section contains over 73,000 grid points, with some grid spacings as small as one meter, while the height and width of the cross section measures about $1.2 \mathrm{~km}$ by $0.7 \mathrm{~km}$. A detailed section of the grid is shown in Figure 12. This is the first mountain-scale flow simulation of YM to include such fine geologic detail. The material properties for these units are based on the best available data. The materials are modeled as fractured rock using the 

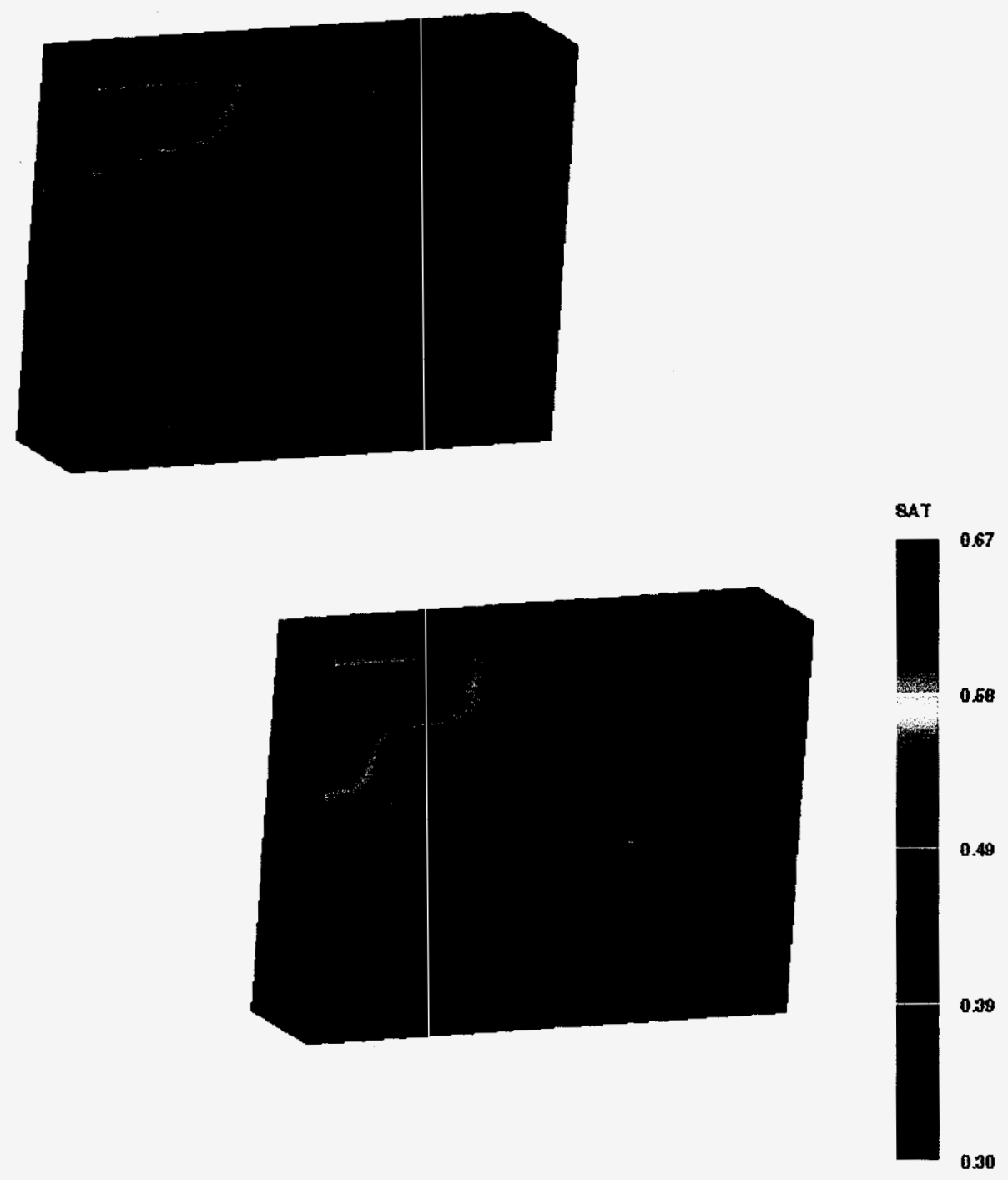

0.67

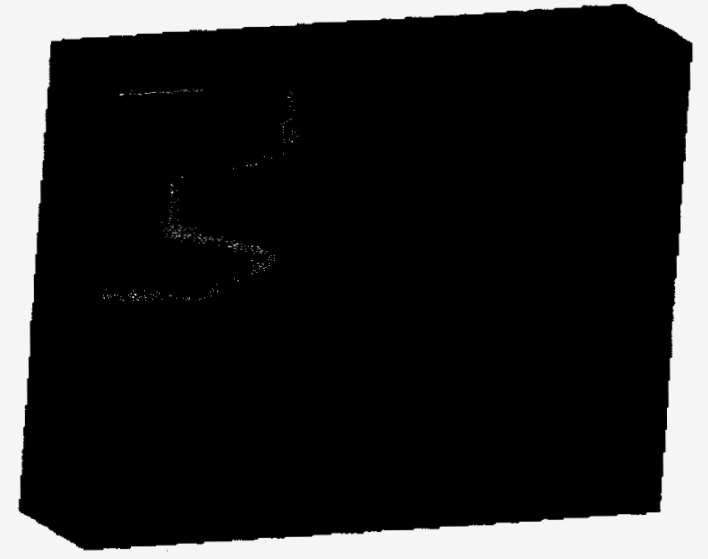

Figure 10 Saturation contours at $t=10,20$, and 30 days. 

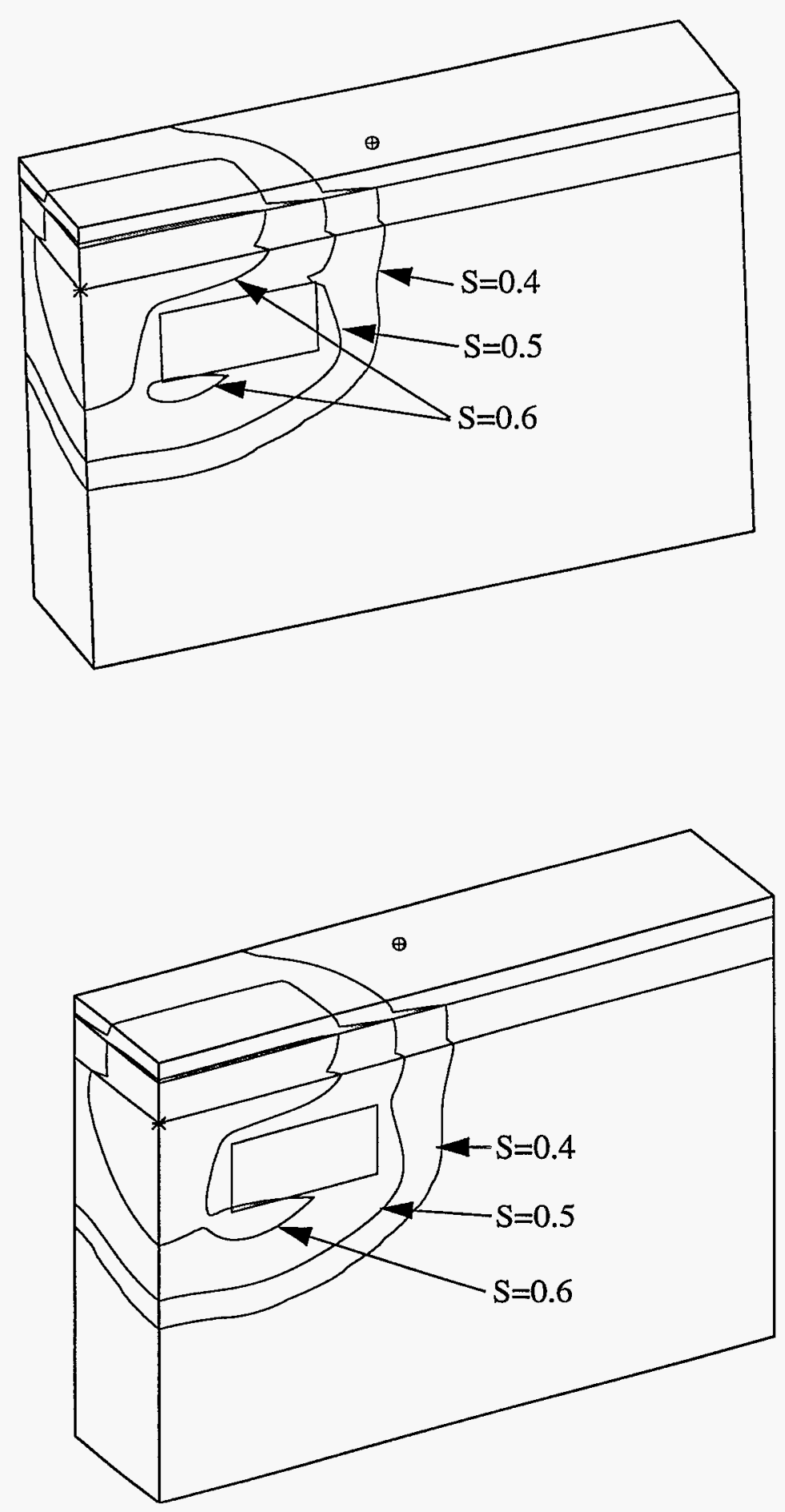

Figure 11 Comparison of saturation contours at $t=30$ days; Coyote II (top) and MPSalsa (bottom). 


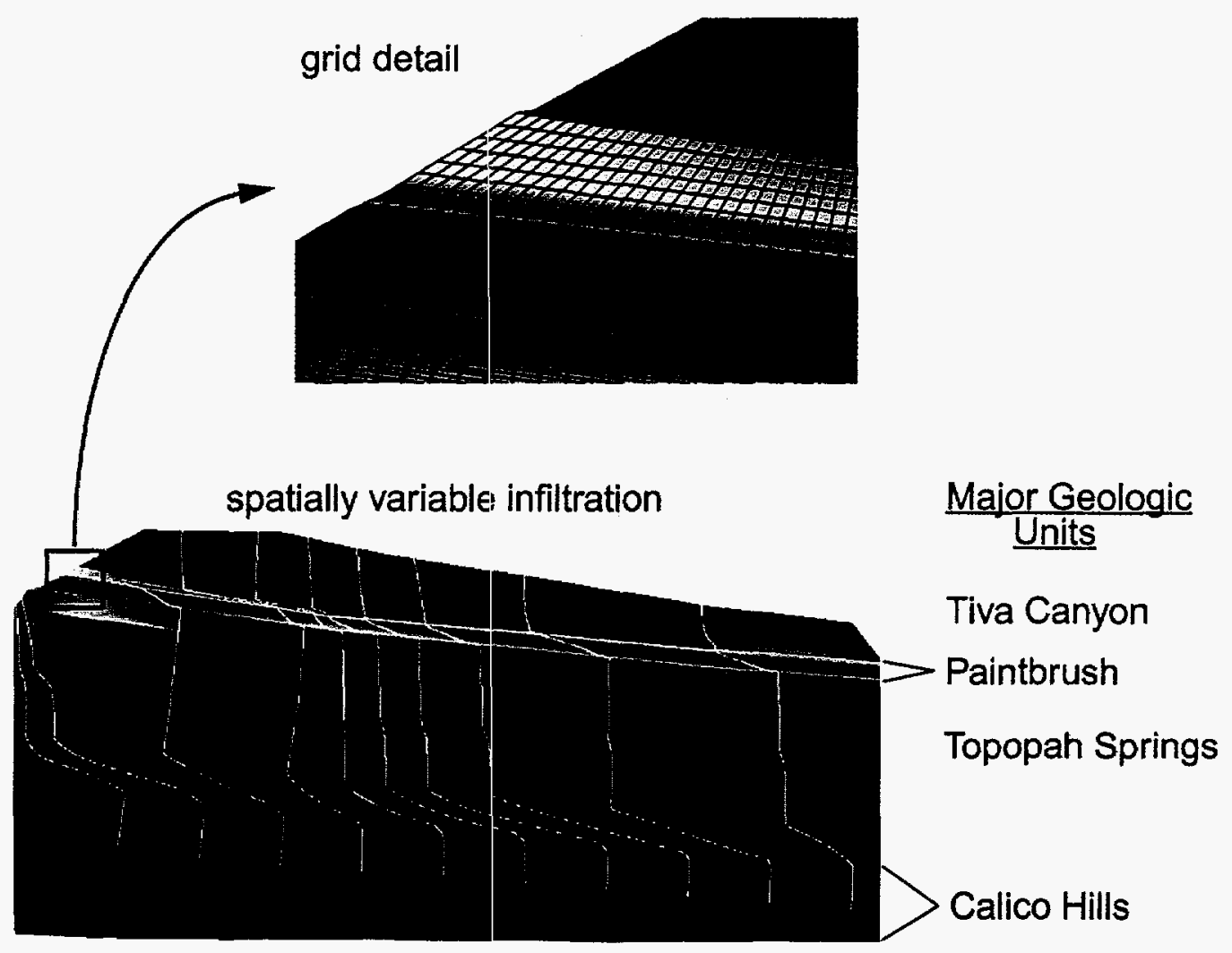

Figure 12 East-west cross section of Yucca Mountain, Nevada. The major hydrogeologic units, which are composed of many sub-units, are labeled. The full grid contains over 73,000 grid points, and resolves many fine geologic details of the cross section, particularly in the Paint Brush unit. Saturations (colors) and flowlines are shown for the case (f) simulation.

so-called equivalent continuum model (ECM) described by Peters and Klavetter (1988) which assumes the fracture and matrix systems are locally in pressure equilibrium. The resulting models for bulk porosity, saturation, and permeability, are given by

$$
\begin{gathered}
\phi_{b}=\phi_{f}+\left(1-\phi_{f}\right) \phi_{m}, \\
\phi_{b} S_{b}=S_{f} \phi_{f}+S_{m}\left(1-\phi_{f}\right) \phi_{m},
\end{gathered}
$$

and

$$
k_{b}=k_{f} \phi_{f}+k_{m}\left(1-\phi_{f}\right),
$$

where $\phi, S$, and $k$ denote porosity, liquid saturation and permeability (product of intrinsic and relative permeability), and subscripts $f, m$, and $b$ denote fracture, matrix and bulk values. Each of the matrix and fracture relative permeability and saturations functions for a particular geologic unit are described by the van Genuchten model given in Eq. (49) and Eq. (50), respectively. Because the saturation functions so described are nonlinear implicit functions of the capillary pressure, the functions were described in tabular form. The 
FORTRAN code for these models was provided by R. R Eaton of SNL. The material data for the models is given in Altman et al., 1996 and will not be repeated here. Suffice it to say these materials, and especially with the ECM, display many orders of magnitude variation in permeability from unit to unit, rendering a highly nonlinear problem which requires the Newton iteration scheme for convergence.

The lateral boundaries were modeled as no-flow boundaries; the lower boundary was modeled as a water table (liquid saturation unity). Three sets of spatially variable infiltration rate distributions, based on measurements at YM, were specified over the upper surface, see Altman et al., 1996. The three infiltration functions, designated case (a) through (c), were arranged in decreasing integrated (total) infiltration. All three are based on observation at similar locations to the present cross section. The objective was to compute a steady solution subject to these boundary conditions. The steady solutions were determined by computing a false transient, starting with an arbitrary initial condition. A uniform initial saturation of $50 \%$ was specified over the domain, except for the layers near the water table, where $80 \%$ saturation was specified to help "smooth" the spatial transition to saturated conditions. Six different cases were run. In addition to the three infiltration distributions, three cases were run with the same infiltration distribution, but with stochastically determined variations in certain geologic units which were likely to effect lateral flow. The idea was to provide conditions, within a realistic statistical range, that might result in significant lateral flow diversion in order to examine its effect on the percolation flux at the repository horizon.

The general trend was that lateral diversion increased with decreasing total infiltration, i.e, case (c), the solution for which is shown in Figure 13(a), displayed the most lateral diversion, as indicated by the lateral displacement of the flowlines ${ }^{4}$ shown. Figure 13 also shows the liquid moisture saturation distribution. The large variation in saturation is due to the strong contrast in material properties for the various hydrostratigraphic units modeled

- this simulation. The saturation variation across the upper surface reflects the spatially rable infiltration flux specified. In addition to the standard cases, a series of cases were simulated in which the fracture matrix properties in adjacent units were perturbed, within the statistical scatter in the flow properties. This was done to illustrate the possible variation in lateral diversion with extreme juxtapositioning of certain units. Figure 13(b) shows the solution for one such variation (so-called case (d)), resulting in a capillary barrier effect in the geologic unit labeled "bt3" in the Paintbrush unit. A close-up view is shown in Figure 14, which also labels the sub-units in the vicinity of the capillary barrier. As indicated, case (d) results in lateral diversion of infiltration entering the unit overlying the PaintBrush unit in the upper west end of the cross section, the so-called Solitario Canyon region. The fine structure is highlighted in this figure, which shows geologic layers, some of which "pinch out," with highly variable saturation distributions. Simulations are compared with saturation measurements in Altman, et al., (1996), which show good agreement. The study demonstrated that the moisture flow in the vicinity of the proposed repository horizon can be highly dependent on the stratigraphic units included in the mod-

4. The "flowlines" are particle paths; the mass flux between two flowlines is constant, but this mass flux is not necessarily the same as between two other flowlines. 


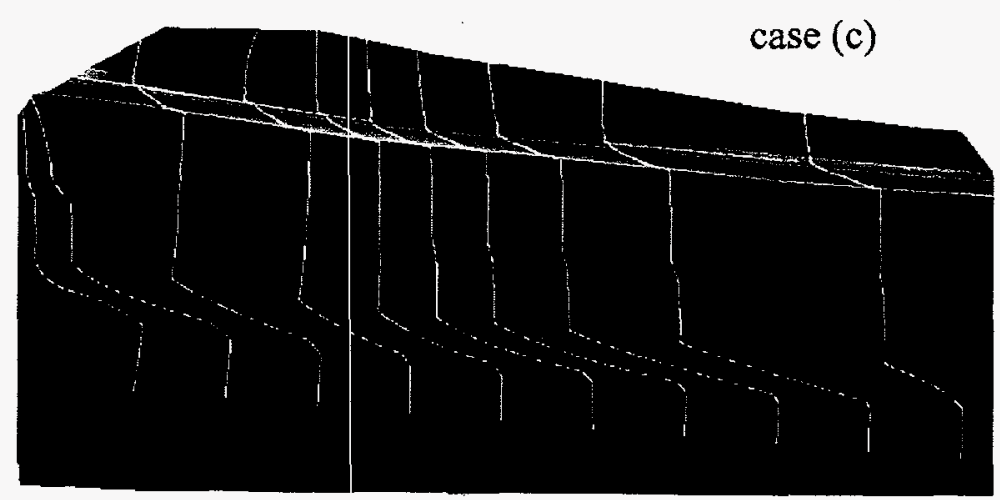

(a)
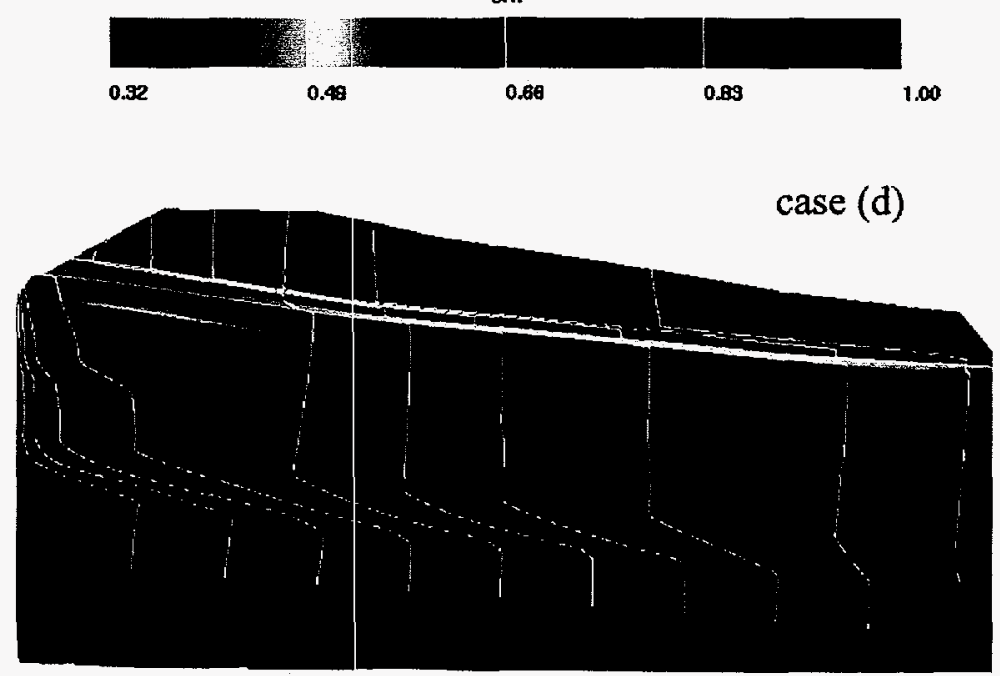

(b)

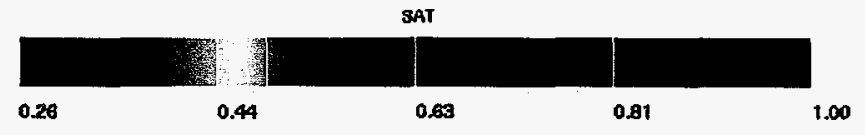

Figure 13 Liquid saturation distribution (color coded) and flow lines in a cross section of Yucca Mountain, Nevada. Significant lateral flow diversion is the result of the large contrast in the fractured material properties.

el. See the aforementioned report by Altman, et al., (1996) for a more complete discussion of this study.

\subsubsection{Hydrothermal transport in fractured rock}

Our final example is also motivated by the YM project. We examine multiphase hydrothermal transport, due to a heat-generating source distribution, in a large scale 3D region modeled after YM. The computational grid, shown in Figure 15, is composed of over 358,000 node points and includes the major hydrostratigraphic units at $\mathrm{YM}$. The waste repository is to be sited in the Topopah Springs unit and is indicated by the fine discretization in the figure, where the heat source is located. The material blocks are shown in Fig. 

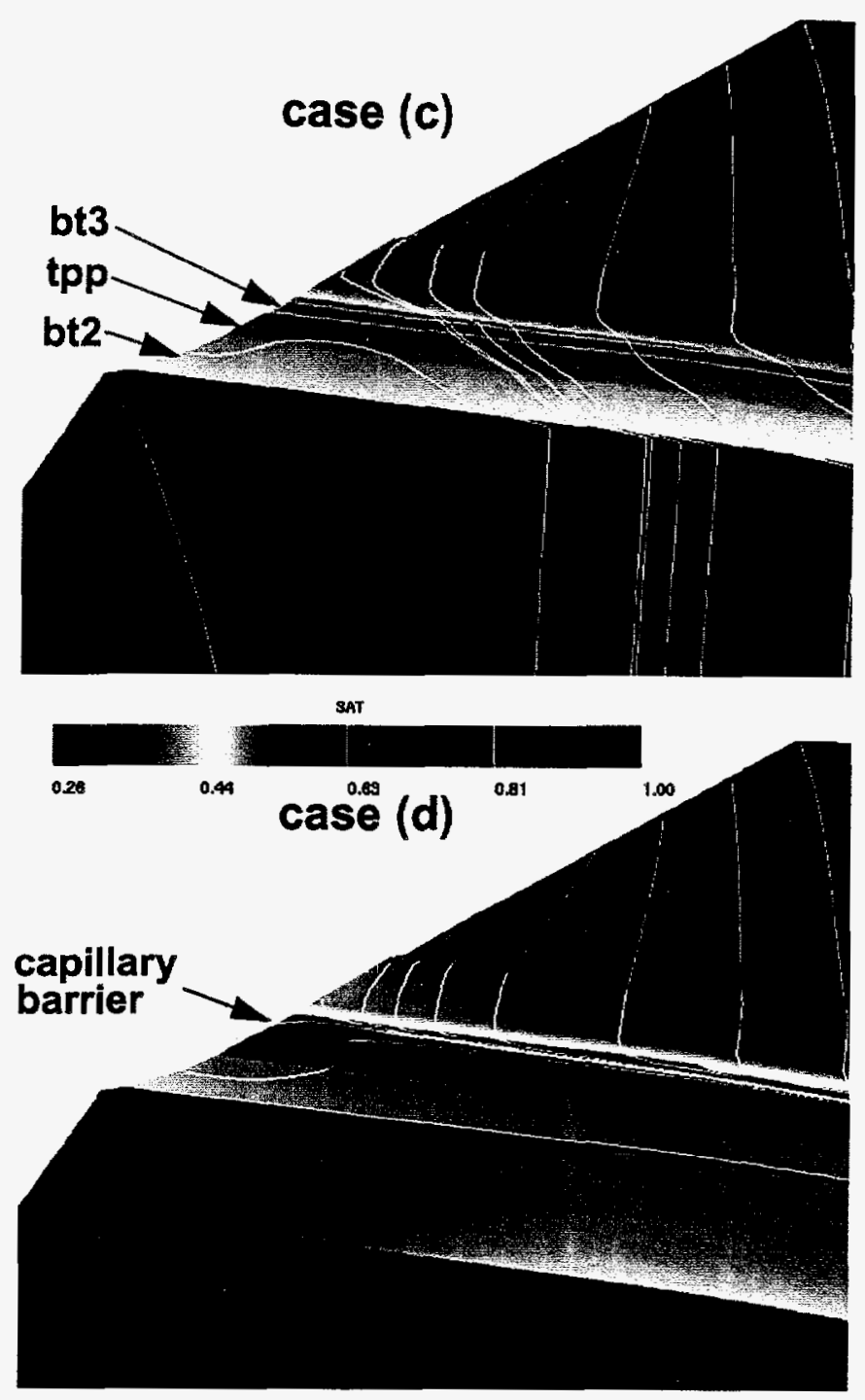

Figure 14 Illustration of a so-called capillary barrier, effected by variation, within the scatter in the data, of fracture/matrix hydrologic properties in the geologic units labelled in the figure. Case (d) results in complete lateral diversion of flow along the geologic unit designated bt3.

16. This hexahedral unstructured finite element mesh was created with the CUBIT (Blacker et al., 1994) mesh generation program. This domain was meant to be similar to YM, but it is not a true representation. The front-facing cross section shown in Figure 15 is in fact representative of YM. However, the full $3 \mathrm{D}$ region was generated by "sweeping" this cross section backward for $3 \mathrm{~km}$ at a vertical inclination of about 5 degrees. Figure 15 includes spatial coordinates of selected points for scale. The entire region measures about $1.6 \mathrm{~km}$ east to west, includes about $0.7 \mathrm{~km}$ above the water table (the lower boundary) and extends $3 \mathrm{~km}$ along its length.

The materials are modeled as composite fractured media using the ECM model introduced in the preceding section. In this case, however, both the gas and liquid phase relative per- 


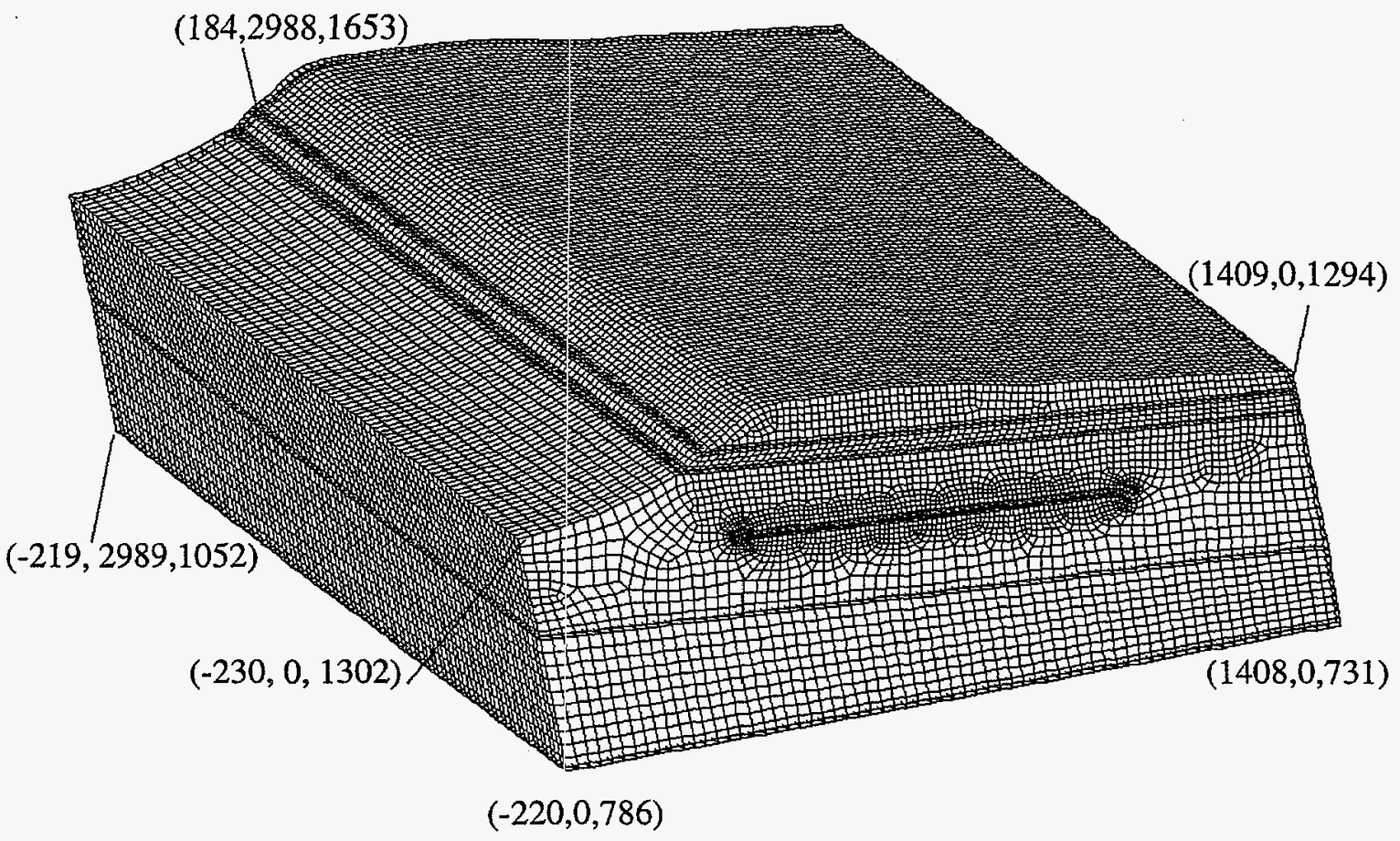

Figure 15 Grid composed of $358,000+$ node points. Selected coordinates $(x, y, z)$ in meters are shown for scale.

meabilities are derived from equations of the form given in Eq. (57). For example the bulk gas permeability is

$$
k k_{r}=k_{f} k_{r, f} \phi_{f}+k_{m} k_{r, m}\left(1-\phi_{f}\right),
$$

where $k_{r, f}$ and $k_{r, m}$ denote the relative permeabilities for the fracture and matrix continua, respectively, and each is given by the functional form $1-k_{r, l}$, in which $k_{r, l}$ is given by the van-Genucthen functional form in Eq. (49). Also, $k_{f}$ and $k_{m}$ are the fracture and matrix intrinsic permeabilities, respectively. The property data for this problem are those specified in the preceding example.

Lateral boundaries are specified as no-flow for all three mass and energy balance equations. The entire lower boundary is modeled as a water table (moisture saturation unity) at 1 atmosphere pressure and a temperature of $20^{\circ} \mathrm{C}$. The upper boundary is also at 1 atmosphere pressure, but at a temperature of $15^{\circ} \mathrm{C}$. An infiltration flux of water at $0.1 \mathrm{~mm} / \mathrm{yr}$ is applied uniformly over the entire upper boundary. These conditions simulate an upper boundary in contact with the atmosphere. The heat source term for the repository was specified as 


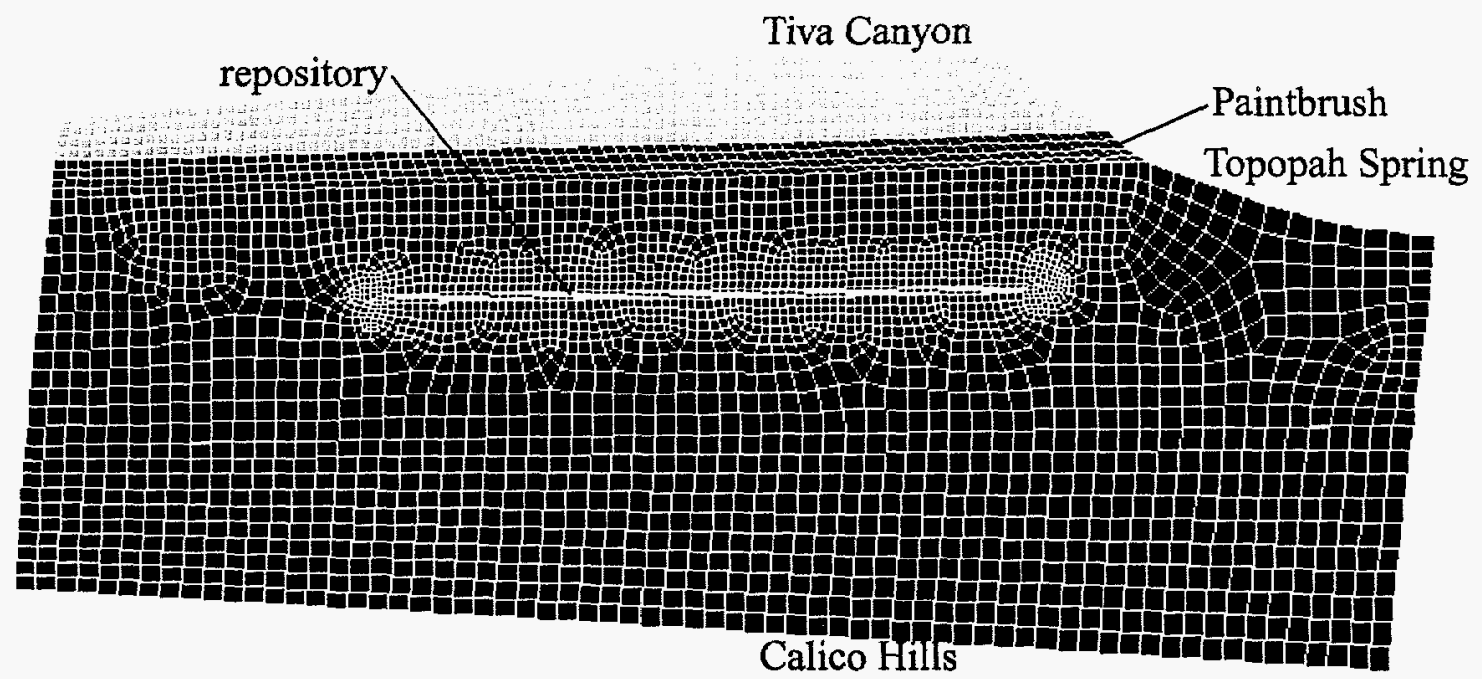

Figure 16 Material blocks and grid on the back side of model. Material blocks are labeled to correspond with YM geologic units.

$$
Q_{e}=Q_{0} \exp \left(-\lambda t-\frac{1}{2}\left(\frac{\left(y-y_{0}\right)}{\sigma}\right)^{2}\right),
$$

with $Q_{0}=0.75 \mathrm{~W} / \mathrm{m}^{2}, \lambda=1 / 63.37 \mathrm{yr}^{-1}, \sigma=494.3 \mathrm{~m}$, and $y_{0}=1500 \mathrm{~m}$. The y-coordinate is perpendicular to the plane shown in Figure 16. The repository block measures $750 \mathrm{~m}$ by $10 \mathrm{~m}$ in the cross section shown in Figure 16.

The simulation was performed in two steps. In order to start with a pre-waste-emplacement condition, a steady state solution was first obtained with no repository heating. This solution provided the initial condition to the next phase of the study which included repository heating.

Figure 17 shows pressure and temperature distributions after 23 years of heat output by the model repository. The temperature is very near to the (thermodynamically) saturated temperature for this pressure. Much of the air has been driven from the repository at this time, see Figure 18. This phenomenon could be important to the question of corrosion of waste containers, a processes whose chemistry may be affected by the absence of air during the hot phase of the repository, before the heat output decays significantly. Some gas pressure build-up above the repository is noted. However, due to the high gas permeability afforded by the fractured medium, the variation in gas pressure is modest. An earlier 2D simulation which modeled materials as matrix-only (unfractured) and with $Q_{0}=1.0 \mathrm{~W} / \mathrm{m}^{2}$ resulted in repository temperatures on the order of $160^{\circ} \mathrm{C}$ and gas pressures of $0.68 \mathrm{MPa}$ after 53 years of heat output. The unfractured matrix permeabilities are ultra-low at $\mathrm{YM}$, on the order of $10^{-18} \mathrm{~m}^{2}$ for the repository horizon, which can trap the evaporated gases in and around the repository resulting in very high pressures and temperatures. 

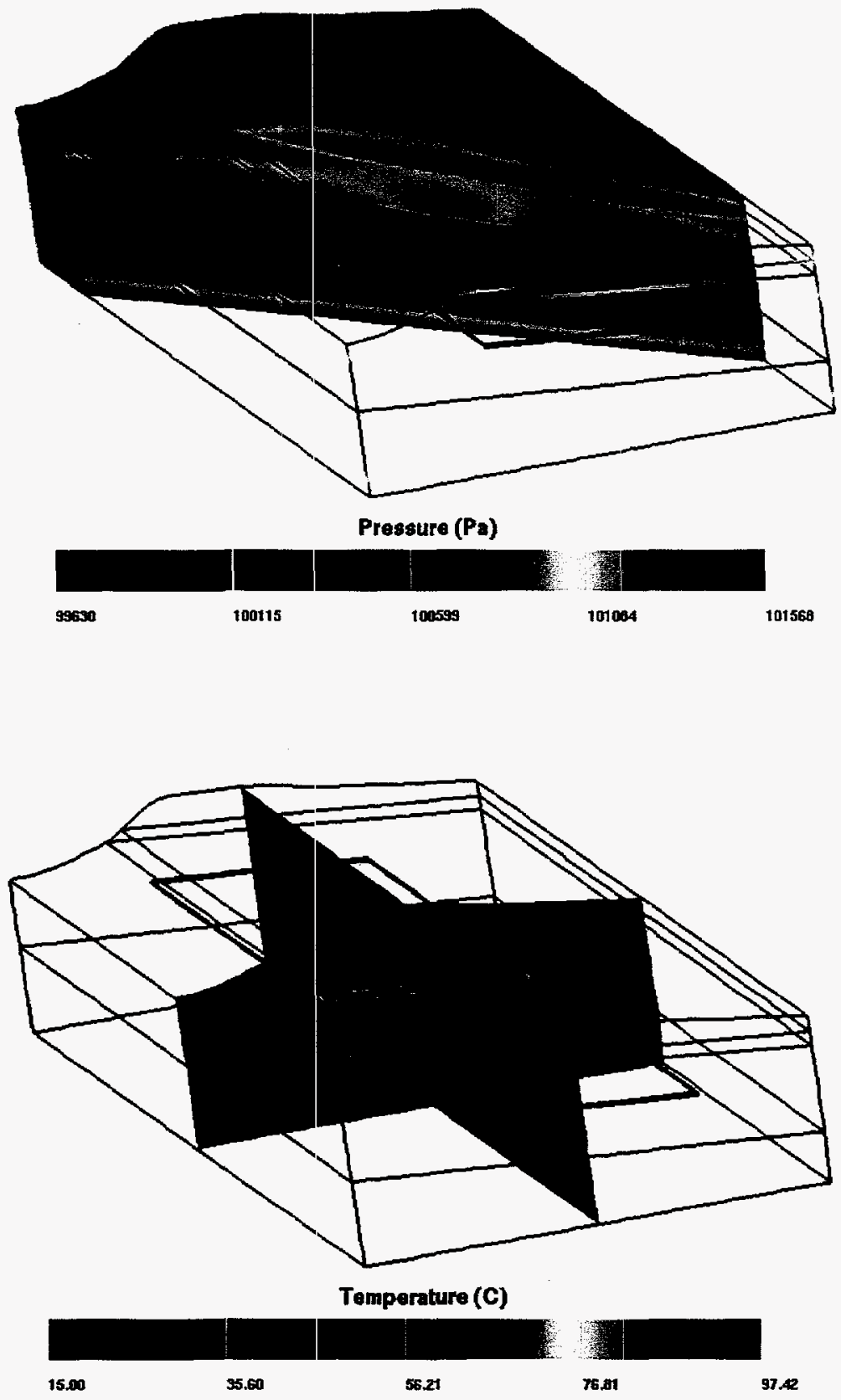

Figure 17 Pressure and temperature distributions at 23 years. Each geologic unit is modeled as a fractured medium, which provides enough gas permeability to negate significant gas pressure build-up. 

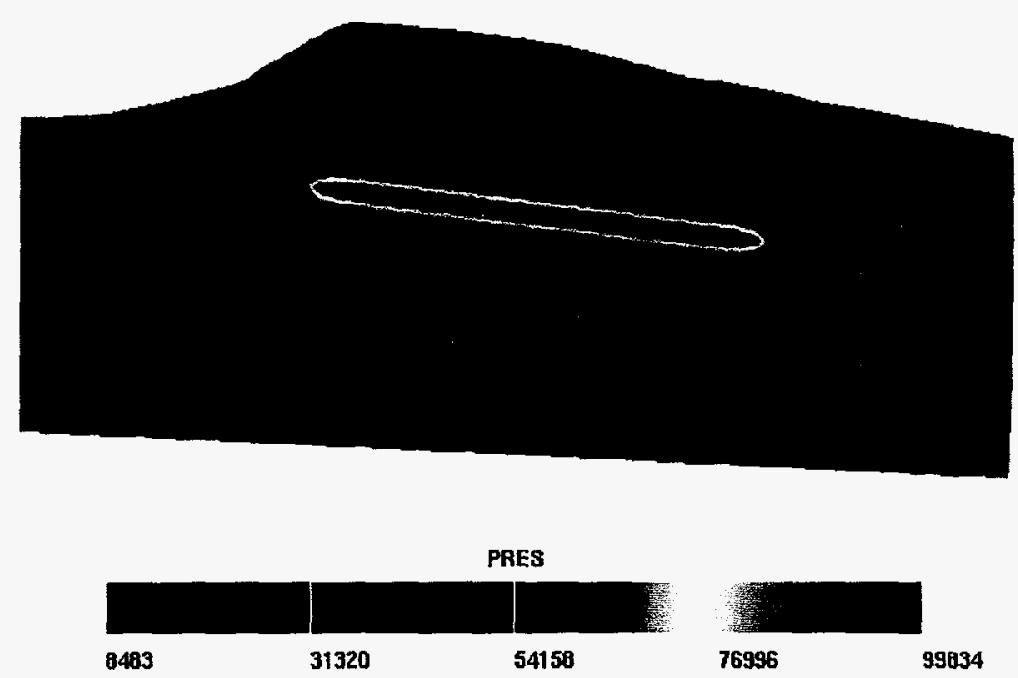

Figure 18 Partial air pressure (Pascals) at 23 years in the plane $y=1500 \mathrm{~m}$. Heatdriven evaporation and subsequent gas transport away from the repository has driven off much of the air, leaving about an $8 \%$ mole fraction at this time.

This simulation, on a mesh consisting of $358,000+$ grid points, requires the solution of a linear system composed of about 1.1 million equations which are solved at each time step. These transient simulations require many 100 s of time steps to simulate flow over many thousands of years. This magnitude of simulation can only be performed with parallel processing computers, as in the current simulator; this is a significant capability. The simulations were run on SNL's 1800-processor Intel Paragon computer; numerical performance studies are discussed in the following section. The capacity of the simulator depends on the size (number of processors and memory per processor) of the parallel computer available. The present example did not utilize the full capacity of the Paragon ${ }^{5}$.

\subsection{Numerical Performance}

\subsubsection{Pre-emplacement infiltration at Yucca Mountain}

A parallel processing performance study was conducted on case (c) of the previously mentioned study. This case displays significant lateral diversion. The study was conducted on the SNL 1800 processor Intel Paragon on 32, 64, 128 and 256 processors. The GMRES solver, with incomplete LU preconditioning (iLU) and no scaling, was used. The steady solution was obtained by solving a transient simulation, using the forward Euler/backward Euler time integrator with automatic time step control, which required 187 time steps and 359 Newton iterations to integrate to a time of 950,000 years, the time-scale for obtaining a steady solution with these material types. The CPU requirements, excluding loading mesh data and file handling, and speedup ratios are shown in Figure 19. Note that the speedup ratio shown is relative to the CPU processing time obtained on 32 processors. The problem is too large to run on one processor of the SNL Paragon. As indicated, the pro-

5. SNL has recently procured an Intel-built 9000 processor teraflop machine. This machine should enable fully transient simulations with roughly $10^{8}$ grid point resolution. 

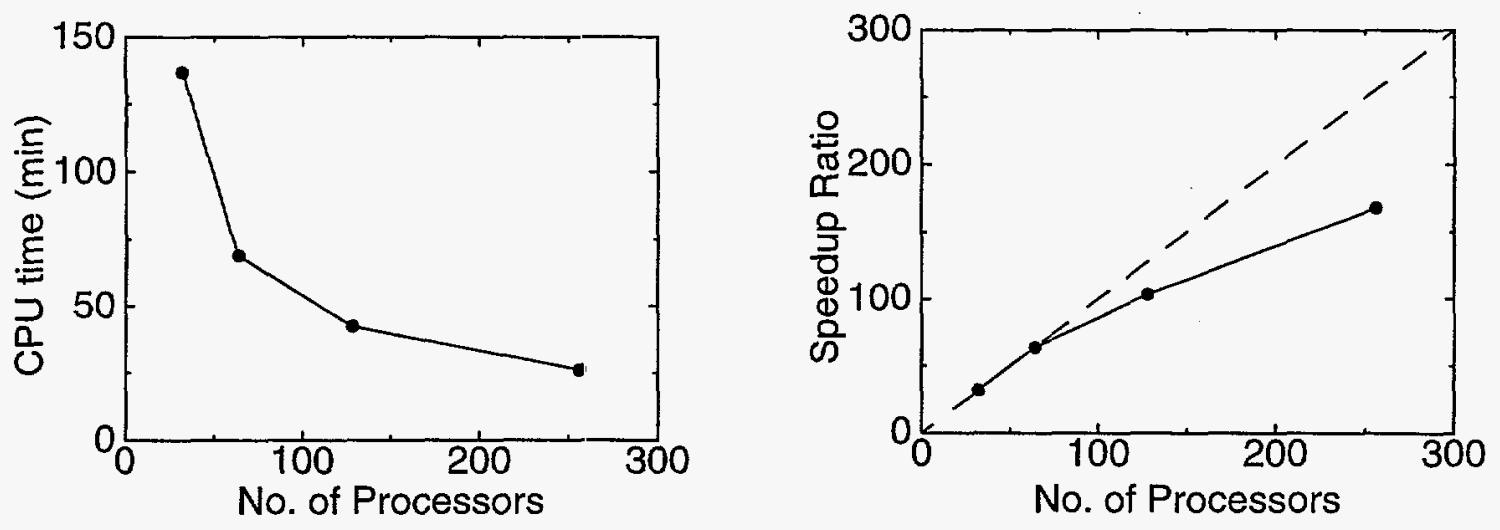

Figure 19 Parallel processing performance data for the 2D Yucca Mountain preemplacement infiltration simulation on a fixed-size grid composed of 73636 node points. Each simulation required 187 time steps, and 359 Newton iterations, demonstrating a fully parallel solver implementation.

cessing time for running the simulation is reduced to under 30 minutes on 256 processors of the Intel Paragon. The same simulation on a single-processor Sun SPARCstation 20 requires on the order of 36 hours, and enough memory to load such a problem. However, the efficiency begins to suffer for 256 processors as communication costs become significant. With 73636 unknowns, the ratio of external-plus-border nodes to internal-plus-border nodes is in the range of $50-55 \%$ for the load balance obtained with Chaco (Hendrickson and Leland, 1993); hence, the communication costs are a large fraction of the processing time. This is inevitable when a fixed-size mesh is partitioned onto an increasing number of processors. On the other hand, the speedup ratio for 64 processors still lies on the theoretical upper bound line. In this case a typical ratio for surface nodes (nodes requiring communication) is about $25 \%$, and yet the communication time doesn't significantly impact the parallel performance. These numbers indicate the message size is also important, typically a fixed cost per byte, beyond a fixed message start-up cost.

\subsubsection{Hydrothermal transport in fractured rock}

This problem was also run on various numbers of processors to assess the parallel performance on the 1800 processor SNL Intel Paragon. In order to reduce the CPU requirements for a mock heat-up and cool down cycle, the heat output was reduced by setting $Q_{0}=0.5$ $\mathrm{W} / \mathrm{m}^{2}$ and the simulation was carried out to 3000 years, starting with the pre-emplacement solution, as described earlier. The GMRES solver, with incomplete iLU preconditioning and row-sum scaling, was used. The iLU preconditioning algorithm was not overlapped over the processors (overlapping is available, but the memory and communication costs are substantial); hence, the number of linear solver iterations required will vary with the number of processors. The row-sum scaling is beneficial, since the solution variables can vary greatly in magnitude.

The total CPU time, excluding loading mesh data and file handling, is shown in Figure 20 as a function of the number of processors. Each simulation, performed with different numbers of processors, required 71 time steps and 125 Newton iterations to integrate the solu- 

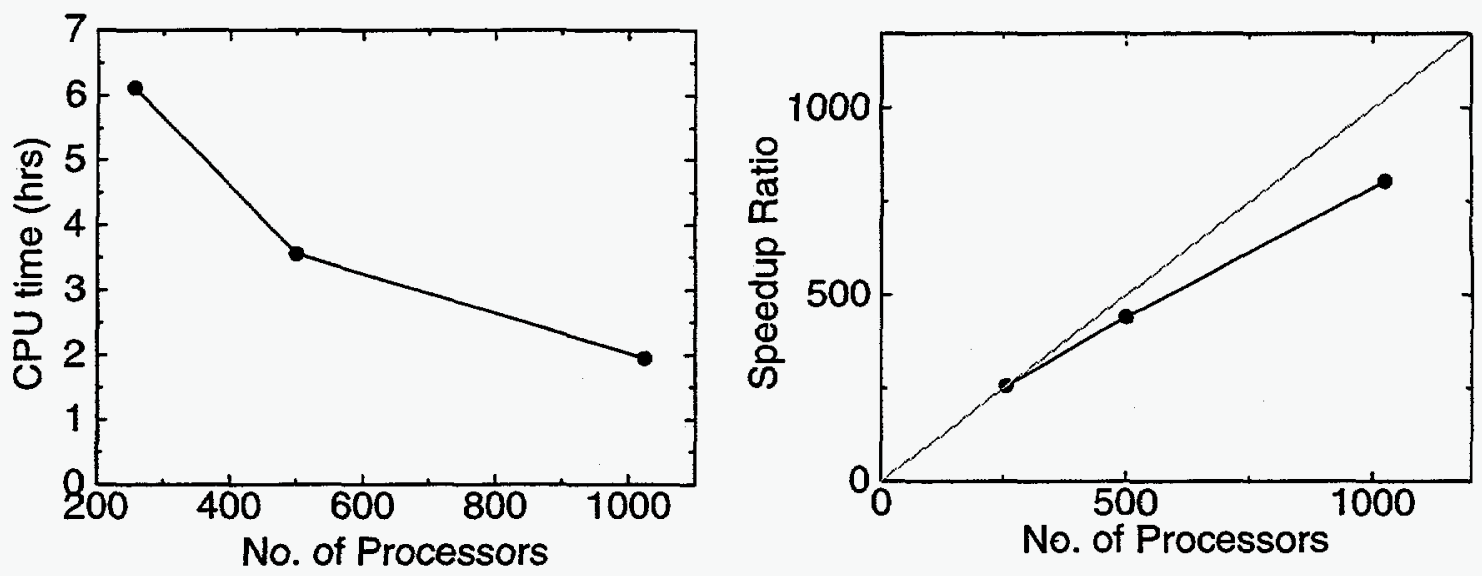

Figure 20 Parallel processing performance data for the 3D hydrothermal simulation. Each simulation required 71 time steps, and 125 Newton iterations, demonstrating a fully parallel solver implementation.

tion out to 3000 yrs. This demonstrates the correct parallel implementation of the solution algorithm. As noted above, the iLU preconditioner was not overlapped on the various processors, and therefore the number of linear solver iterations varies with the number of processors. For the present simulation, 9023, 9841, and 10103 total linear iterations were necessary on 256, 500 and 1024 processors, respectively. The speedup ratio shown was defined as

$$
\frac{256 \bullet T_{256}}{T_{p}}
$$

where $T_{p}$ denotes the CPU time on $p$ processors. The numerator estimates the CPU time on a single processor based on the CPU time for 256 processors. This estimate is not expected to be accurate (relative to the speedup ratio based on execution time on one processor), and neither is the speedup ratio for 256 processors shown on Figure 20. The current problem is far too large to fit on a single processor of the Intel Paragon. It in fact didn't fit on as few as 200 processors (The limitation was the GMRES solver, with 32 Krylov subspace vectors requested). Utilizing 1024 processors, the relative efficiency is about $79 \%$, a good value for an unstructured grid parallel algorithm.

Figure 21 compares the time-step history between the second-order Adams-Bashforth/ trapezoid-rule (ABT) integrator and the first-order forward-Eulet/backward-Euler (FEBE) scheme. The former scheme performs the integration to 3000 years in 48 time steps (77 Newton iterations) versus 71 steps (125 Newton iterations) for the Euler scheme. On this problem, the second-order method performs the integration in about $59 \%$ of the time required by the first order method. Note that although the ABT method increased the time step sizes quickest, the time step size for the FEBE method has "caught-up" at the end of the simulation. 


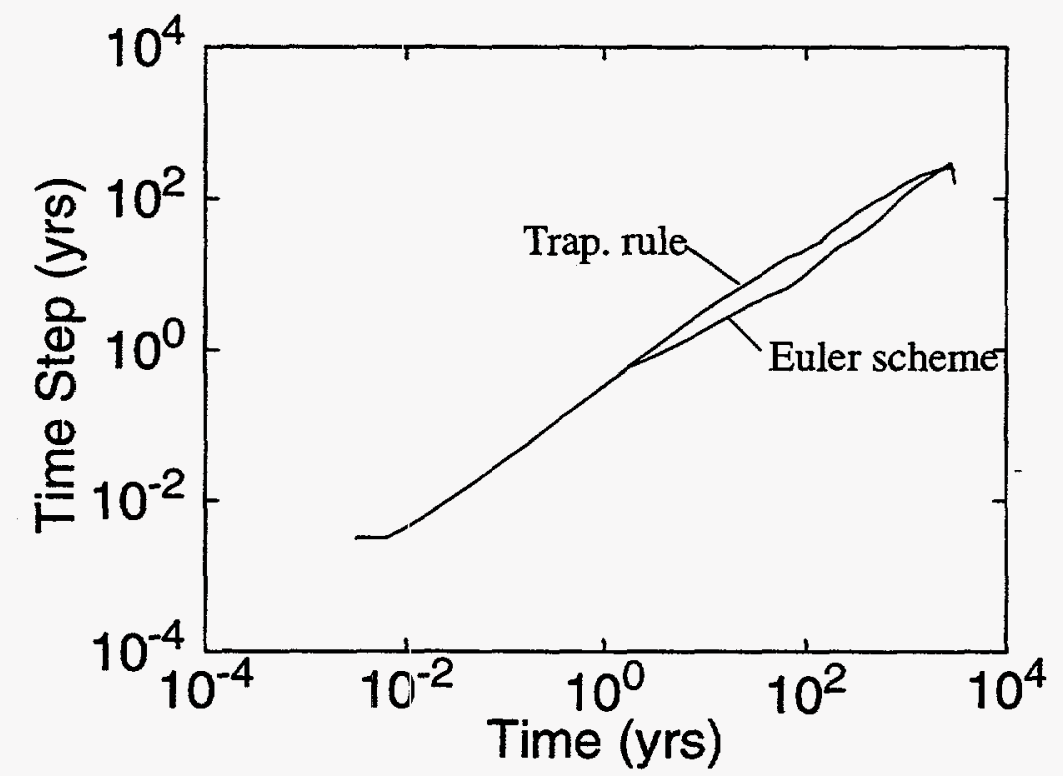

Figure 21 Time step history comparison between the first-order and second order time integration methods.

\subsubsection{Three-dimensional flow in unsaturated porous media}

To demonstrate the code's capability to execute in parallel on multiple workstations using the Message Passing Interface (MPI), the three-dimensional problem described in Section 5.1.4 was executed on one, two and four Sun SPARCstation20 workstations. The GMRES solver, with incomplete LU preconditioning and no scaling, was used. The forward Euler/ backward Euler integrator with automatic time step control was used for the transient simulation which required 80 time steps and 164 Newton iterations to integrate to a time of 30 days. The CPU requirements, including loading mesh data and file handling, and speedup ratios are shown in Figure 22 . Note that using two workstations yields a speedup ratio of 1.98 , very near the theoretical limit of 2 . With four workstations, the communications costs bring the speedup ratio down from a theoretical value of 4 to 3.75 . This $94 \%$ efficiency is still very good for performing communications over ethernet, demonstrating that existing distributed computing assets can still be used effectively to perform parallel calculations with the simulator developed in this project.

\section{Concluding Remarks}

\subsection{Summary}

The major objectives of this LDRD-sponsored project were to initiate a research program in multiphase multicomponent subsurface transport, and to develop state-of-the-art computational tools for numerical simulation of such problems. These goals were successfully achieved in this project. This project: has produced a strong foundation for continued re- 

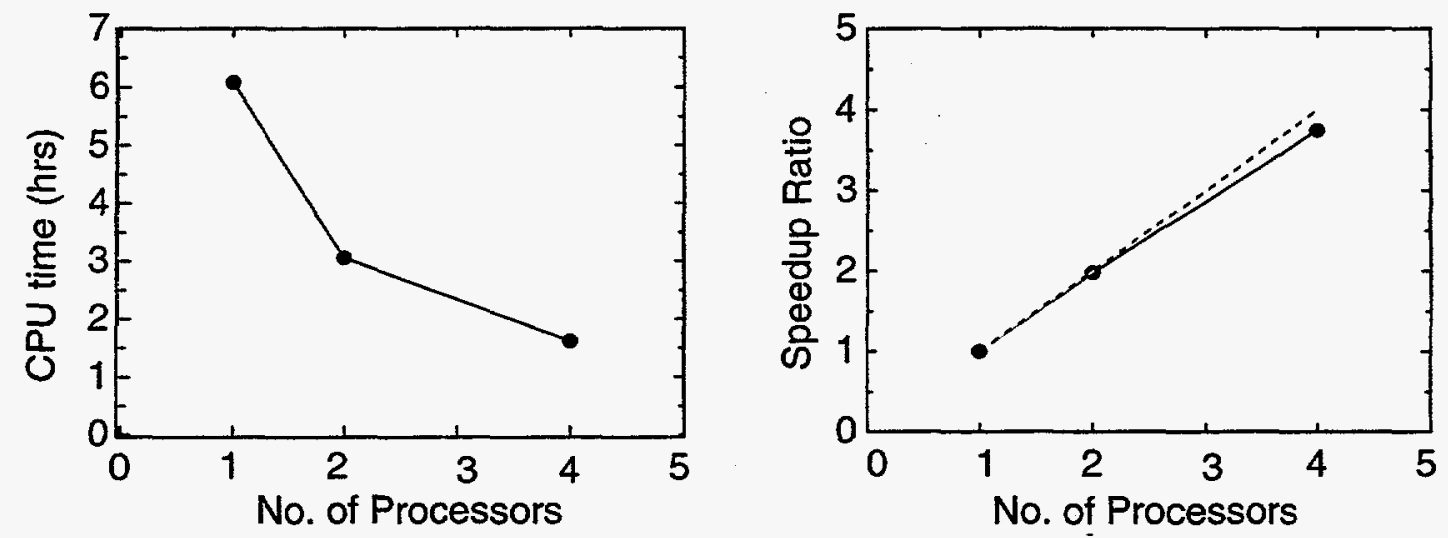

Figure 22 Parallel processing performance data on a network of workstations communicating over ethernet via MPI. The problem is for patch infiltration into a $3 \mathrm{D}$ unsaturated heterogeneous medium on a fixed-size grid composed of 21758 node points.

search into project-funded subsurface transport problems. The numerical platform in particular provides an excellent base for developing simulation capability in areas of current interest to SNL including, nuclear subsurface waste disposal and cleanup, groundwater availability and contamination studies, fuel-spill fires for weapon and transportation safety, heat-driven foam decomposition in weapon components, and DNAPL transport and remediation, to name just a few.

The major accomplishment was the development of a general-purpose 3D unstructured grid, two-phase, two-component subsurface transport, finite element simulator. The MP algorithm is scalable. The code has been applied to an SNL project, where high-resolution simulations were required. This simulator can be run on the Intel Paragon, IBM SP-2, as well as on a network of workstations; it is not necessary to have access to an MP machine. However, the code can be described as an "beta" version, fully capable of the physics described in the foregoing, but requiring some additional development of the user interface. The performance results clearly show the potential for this general purpose MP capability. In particular, the previously discussed applications demonstrate that 3D, large-scale multiphase simulations with high resolution of geologic strata are feasible, and moreover can now be performed routinely on the SNL Intel Paragon. However, the parallel processing capabilities can also be accessed on (ubiquitous) networked systems using MPI. This enables 3D high-resolution parallel processing simulations to be performed on common networked systems.

\subsection{Recommendations on Future Directions}

The following enumerates some areas for development which could provide a significant cost/benefit for invested resources.

1. The ability to couple the code with geostatistical property simulators should be implemented, to enable statistical analysis of the impact of spatially correlated heterogeneity and uncertainty analysis. A general interface for specifying 
heterogeneous data has recently been implemented. It allows the user to specify heterogeneous data not only for typical parameters, such as permeability and porosity, but also for user-specified parameters appearing in transport models, such as relative permeability functions, etc.

2. The ability to simulate transport of dilute species would enable modeling of conservative and non-conservative (e.g., radioactive) contaminants, and the transport of organics in quantities below their aqueous solubility limits. This would allow the simulation and design of many existing and emerging remediation technologies. The present code already includes the capability to include auxiliary transport equations, and so this capability would require few architectural modifications. However, prior experience (Martinez, 1985) indicates high-Peclet number convection schemes may be warranted, e.g., along the lines of the streamline upwind Petrov-Galerkin (SUPG) method (Brooks and Hughes, 1982) or the Galerkin least-squares (GLS) technique (Hughes et al., 1989).

3. The code's applicability to the understanding and remediation of non-aqueous-phaseliquids (NAPLs) in concentrations greater than their aqueous solubility limits could be greatly enhanced by implementing a three-phase, multicomponent capability. The treatment of multiphase, multicomponent phase equilibria (Walas, 1985; Callen, 1985) would require a significant effort. A formulation for this type of problem was completed in this project (Martinez, 1995b) utilizing a partition-coefficient approach to phase equilibria, which was also researched under this project (Ho, 1995a, 1995b). A fully compositional, Gibbs free-energy minimization approach to phase equilibria coupled with a three-phase flow model would offer the most general simulation capability. However, this would entail a significant development effort, on the order of 2-3 FTEs for 2 years. 


\section{References}

Altman, S. J., S. Mishra, C. Li, J. T. Birkholzer, J. E. Houseworth, C. F. Tsang, S. A. McKenna, Y. W. Tsang, R. R. Eaton, V. C. Tidwell, J. S. Y. Wang, B. S. RamaRao, M. J. Martinez, P. B. Davies, 1996, Multi-scale modeling to evaluate scaling issues, percolation flux and other processes for PA recommendations, Level E Milestone T6540, Yucca Mountain Project, Las Vegas, NV, September, 1996.

Arbogast, T., M. F. Wheeler, and I. Yotov, 1994, Logically rectangular mixed methods for groundwater flow and transport on general geometry, Comp. Meths. in Water Resources X, Vol 1., (A. Peters et al., eds. ) Kluwer Academic Publishers, Dordrecht, The Netherlands, 149-156.

Ashby, S. F., R. D. Falgout, S. G. Smith, A. F. B. Tompson, 1994, Modeling groundwater flow on MPPs, in Proc. 1993 Scalable Parallel Libraries Conf., IEEE Computer Society Press, 17-25.

Aziz, K., and Settari, A., 1979, Petroleum Reservoir Simulation, Applied Science Publishers, Ltd., London, 476 pp.

Bear, J., 1972, Dynamics of Fluids in Porous Media, American Elsevier, New York, 764 pp.

Bird, R. B., W. E. Stewart, and E. N. Lightfoot, 1960, Transport Phenomena, Wiley, New York, $780 \mathrm{pp}$.

Blacker, T. D., W. J. Bohnhoff, T. L. Edwards, J. R. Hipp, R. R. Lober, S. A Mitchell, G. D. Sjaardema, T. J. Tautges, T. L. Wilson, W. R. Oakes, S. Benzley, J. C. Clements, L. Lopez-Buriek, S. Parker, M. Whitely, D. White and E. Trimble, 1994, Sandia National Laboratories Tech. Rept., SAND94-1100, Sandia National Laboratories, Albuquerque, NM, 180 pp.

Brooks, A. N., and T. J. R. Hughes, 1982, Steam-line upwind Petrov-Galerkin formulation for convection dominated flow with particular emphasis on the incompressible NavierStokes equations, Comp. Meths. Appl. Mech. Engrg., 32, pp. 199-259.

Callen, H. B., 1985, Thermodynamics, 2nd. Ed., John Wiley \& Sons, New York, 493 pp.

Coats, K. H., 1978, A highly implicit steamflood model, Soc. of Pet. Eng. J., 18 (5), $369-$ 383.

Faita, R. W., K. Pruess, I. Javandel, and P. A. Witherspoon, 1990, Numerical modeling of steam injection for the removal of nonaqueous phase liquids from the subsurface. 1 . Numerical formulation, LBL-29615, Lawrence Berkeley Laboratory, Berkeley, CA, 70 pp.

Foster, I., 1995, Designing and Building Parallel Programs, Addison-Wesley Publishing Company, Menlo Park, CA, 390 pp. 
Gartling, D. K., and R. E. Hogan, 1994, Coyote II - A finite element computer program for nonlinear heat conduction problems, Sandia National Laboratories Tech. Rept., SAND941173, Sandia National Laboratories, Albuquerque, NM, 62 pp.

Gill, P. E., W. Murray, and M. H. Wright, 1981, Practical Optimization, Academic Press, New York, 401 pp.

Gresho, P. M., R. L., Lee, and R. L. Sani, 1980, On the time-dependent solution of the incompressible Navier-Stokes equations in two and three-dimensions, Recent Advances in Numerical Methods in Fluids, Volume 1, Pineridge Press Ltd., Swansea, U. K., 27-81.

Gropp, W., E. Lusk, and A. Skjellurn, 1995, Using MPI, MIT Press, Cambridge, 307 pp.

Hassanizadeh, M. S., and W. G. Gray, 1990, Mechanics and thermodynamics of multiphase flow in porous media including interphase boundaries, Adv. Water Resources, 13(4), pp., 169-186.

Hendrickson, B., and R. Leland, 1993, The Chaco user's guide, SAND93-2339, Sandia National Laboratories, Albuquerque, NM, 22 pp.

Ho, C. K., 1995a, Numerical simulations of multicomponent evaporation and gas-phase transport experiments using M2NOTS, Proc. of Multiphase Flow in Waste Man. and Environ. Rest., 1995 ASME/AIChE Natl. Heat Transfer Conf., Portland, Oregon, August 5-9.

Ho, C. K., 1995b, Multicomponent three-phase equilibria, Sandia National Laboratories Tech. Rept. SAND95-1063, Sandia National Laboratories, Albuquerque, NM, 26 pp.

Hopkins, P. L., 1990, COVE 2A benchmarking calculations using LLUVIA, Sandia National Laboratories Tech. Rept., SAND88-2511, Sandia National Laboratories, Albuquerque, NM.

Hopkins, P. L., and R. R. Eaton, 1990, LLUVIA: A program for one-dimensional steadystate flow through partially saturated porous media, SAND88-0558, Sandia National Laboratories, Albuquerque, NM.

Hughes, T. J. R., 1987, The Finite Element Method, Prentice-Hall, inc., Englewood Cliffs, NJ, 803 pp.

Hughes, T. J. K., L. P. Franca, and G. M. Hulbert, 1989, A new finite element formulation for computational fluid dynamics: VIII. The Galerkin/least-squares method for advectivediffusive equations, Comp. Meths. Appl. Mech. Engrg., 73, pp. 173-189.

Hutchinson, S. A., J. N. Shadid and R. S. Tuminaro, 1995, Aztec user's guide, Version 1.0, Sandia National Laboratories Tech. Rept., SAND95-1559, Sandia National Laboratories, Albuquerque, NM, 40 pp.

Lake, L. W., 1989, Enhanced Oil Recovery, Prentice-Hall, New Jersey, 550 pp. 
Martinez, M. J., 1985, FEMTRAN -- A finite element computer program for simulating radionuclide transport through porous media, Sandia National Laboratories Tech. Rept., SAND84-0747, Sandia National Laboratories, Albuquerque, NM, 82 pp.

Martinez, M. J., 1988, Capillary-driven flow in a fracture located in a porous medium, Sandia National Laboratories Tech. Rept. SAND84-1697, Sandia National Laboratories, Albuquerque, NM.

Martinez, M. J., and K. S. Udell, 1990, Axisymmetric creeping motion of drops through circular tubes, J. Fluid Mech., 210, 565-591.

Martinez, M. J., and D. F. McTigue, 1991, A boundary integral equation method for steady two-dimensional flow in partially saturated media, Sandia National Laboratories Tech. Rept., SAND90-0253, Sandia National Laboratories, Albuquerque, NM, 58 pp.

Martinez, M. J. , 1995a, Formulation and numerical analysis of nonisothermal multiphase flow in porous media, Sandia National Laboratories Tech. Rept., SAND94-0379, Sandia National Laboratories, Albuquerque, NM, 28 pp.

Martinez, M. J. , 1995b, Mathematical and numerical formulation of nonisothermal multicomponent three-phase flow in porous media, Sandia National Laboratories Tech. Rept., SAND95-1247, Sandia National Laboratories, Albuquerque, NM, 27 pp.

Martinez, M. J., P. L. Hopkins, and M. W. Glass, 1995, Modeling flow in porous media on parallel computers, First Biennial Tri-Laboratory Engineering Conference, Pleasanton, CA, Oct. 31 - Nov 2, 1995.

Martinez, M. J., and R. R. Eaton, 1996, Assessment of multiphase flow codes for environmental remediation problems, paper presented at the FEHM Workshop, Los Alamos National Laboratory, Los Alamos, NM, Feb. 21-23.

Martinez, M. J., and R. H. Nilson, 1997, Estimates of barometric pumping of moisture through unsaturated fractured rock, submitted to Water Resources Research.

Mualem, Y., 1976, A new model for predicting the hydraulic conductivity of unsaturated porous materials, Water Resources Research, 12 (3), 513-522.

Nilson, R. H., and M. J. Martinez, 1994, Barometric pumping of contaminated gases through unsaturated fractured rock, Chapter 21, Total System Performance Assessment for Yucca Mountain, TSPA-1993, Sandia National Laboratories Tech. Rept., SAND93-2675, Sandia National Laboratories, Albuquerque, NM.

Peaceman, D. W., 1977, Fundamentals of Numerical Reservoir Simulation, Elsevier, New York.

1995 Partnership in Computational Sciences (PICS) Research Proposal, 1995, website address: http://www.isc.tamu.edu/PICS, Institute for Scientific Computing, Texas A\&M University. 
Press, W. H., Teukolsky, S. A., Vetterling, W. T., and Flannery, B. P., Numerical Recipes: The Art of Scientific Computing, Cambridge University Press, Cambridge, 963 pp.

Pruess, K., 1987, TOUGH user's guide, LBL-20700 (NUREG/CR-4645), Lawrence Berkeley Laboratory, Berkeley, CA 78 pp.

Raviart, P. A. and J. M. Thomas, 1977, A mixed finite element method for second order elliptic problems, Mathematical Aspects of Finite Element Methods, Lecture nodes in Mathematics, (I. Galligani and E. Magenes), Springer-Verlag, New York, 292-315.

Reid, R. C., J. M. Prausnitz, and B. E. Poling, 1987, The Properties of Gases and Liquids, 4th. Ed., McGraw-Hill, New York, 741 pp.

Scheidegger, A. E., 1974, The Physics of Flow Through Porous Media, University of Toronto Press, Toronto, $353 \mathrm{pp}$.

Shadid, J. N., H. K. Moffat, S. A. Hutchinson, G. L. Hennigan, K. D. Devine, and A. G. Salinger, 1996, MPSALSA, A finite element computer program for reacting flow problems, Part 1 - Theoretical development, Sandia National Laboratories Tech. Rept., SAND95-2752, Sandia National Laboratories, Albuquerque, NM, 81 pp.

Stone, H. L, 1973, Estimation of three-phase relative permeability and residual oil data, $J$. Can. Petrol. Tech., 12(4), 53-61.

Somerton, W. H., J. A. Keese, and S. L. Chu, 1974, Thermal behavior of unconsolidated oil sands, SPE J., 14(5), October.

Tompson, A. F. B., R. D. Falgout, S. G. Smith, W. J. Bosl, and S. F. Ashby, 1996, Analysis of subsurface contaminant migration and remediation using high performance computing, http://www-ep.es.llnl.gov/www-ep/esd, Lawrence Livermore National Laboratories, Livermore, CA.

Udell, K. S., and J. S. Fitch, 1985, Heat and mass transfer in capillary porous media considering evaporation, condensation, and non-condensible gas effects, presented at the $23 \mathrm{rd}$ ASME/AICHE National Heat Transfer Conference, Denver CO.

van der Vorst; H., 1990, Iterative methods for the solution of large systems of equations on supercomputers, Adv. Water Resources, 13 (3), 137-146.

van Genuchten, R., 1978, Calculating the Unsaturated Hydraulic Conductivity with a New Ciosed Form Analytical Model, Water Resources Bulletin, Princeton University Press, Princeton University, Princeton, Nj.

Vargaftik, N. B., 1975, Tables of the Thermophysical Properties of Liquids and Gases, 2nd Ed., John Wiley and Sons, New York.

Walas, S. M., 1985, Phase Equilibria in Chemical Engineering, Butterworth Publishers, Boston, $671 \mathrm{pp}$. 
Williams, F. A., 1985, Combustion Theory, 2nd Ed., Benjamin/Cummings Publ. Co. Inc., Menlo Park, CA, 680 pp.

Zyvoloski, G. A., B. A. Robinson, Z. V. Dash, and L. L. Trease, 1995, Models and methods summary for the FEHMN application, LA-UR-94-3787, Los Alamos National Laboratory report, Los Alamos NM, 60 pp. 


\section{DISTRIBUTION:}

Rachid Ababou

Commissariat a l'Energie Atomique Saclay

DRN/DMT/SEMT/TTMF

91191 Gifsur-Yvette Cedex

FRANCE

Dr. Steven Ashby

Lawrence Livermore Natl. Lab.

M/S L-561

P. O. Box 808

Livermore, CA 94551-0808

Dr. Richard A. Cairncross

Mech. Eng. Dept.

University of Delaware

313 Spencer Laboratory

Newark, DE 19716-3140

Dr. I. Carpenter

Environmental Applications

Cray Research/SGI

655E Lone Oak Dr.

Eagan, MN 55121

Prof. M. A. Celia

C-319F, E-quad

Princeton University

Princeton, NJ 08544

Prof. Clint Dawson

Dept. of Engineering Mechanics

University of Texas at Austin

Austin, TX 78712

D. A. Dreyfus (RW-1)

Director

OCRWM

US Dept. of Encrgy

1000 Independence Ave. SW

Washington, DC 20585

2 DOE

Attn: E. Smistad, A. Van Luik

101 Convention Center Drive 
P200-219

Las Vegas, NV 89109

Prof. Jim Douglas Jr.

Center for Applied Mathematics

Purdue University

West Lafayette, IN 47907

Dr. R. R. Eaton

10428 Hendrix NE

Albuquerque, NM 87111

Prof. R. E. Ewing

Inst. for Scientific Computation

Texas A\&M University

College Station, TX 77843-3404

Prof. W. G. Gray

Dept. of Civil Eng. and Geol. Sci.

University of Notre Dame

Notre Dame, IN 46556-0767

R. Green

CNWRA

6220 Culebra Road

San Antonio, TX 78238-5166

Dr. J. T. McCord

Daniel B. Stephens \& Assoc.

6020 Academy Rd. NE 87109

Prof. C. T. Miller

Dept. of Environmental Sciences

University of North Carolina

Chapei Hill, NC 27599

2 Lawrence Berkeley Laboratory

Attn: K. Pruess, G. S. Bodvarsson

Earth Sciences Div.

1 Cyclotron Road, MS 50E

Berkeley, CA 94720

R. W. Nelson

INTERA

101 Convention Center Drive

Suite 540 
Las Vegas, NV 89109

M. Reeves

INTERA

6850 Austin Center Blvd.

Suite 30

Austin, TX 78731

Prof. T. F. Russell

Dept. of Mathematics

Univ. of Colorado, Denver

P. O. Box 173364, Campus Box 170

Denver, CO 80217-3364

L. D. Stewart

Praxis Environmental Technologies, Inc.

1440 Rollins Road

Burlingame, CA 94010

J. S. Stuckless, Chief

Geologic Studies Program

MS 425

Yucca Mountain Project Branch

US Geological Survey

P. O. Box 25046

Denver, CO 80225

Dr. Frank Titus

State Engineer Tech. Advisory Comm.

2864 Tramway Cir. NE

Albuquerque, NM 87122

2 University of California, Berkeley

Attn: K. S. Udell, A. C. Fernandez-Pello

Department of Mechanical Engineering

Berkeley, CA 94720

University of Washington

Attn: D. F. McTigue

Department of Geological Sciences, AJ-20

Seattle, WA 98195

Prof. Mary F. Wheeler

Director, Center for Subsurface Modeling

University of Texas at Austin

Taylor Hall 2.400 / UT Mail Code C0200 
Austin, TX 78712

2 U.S. Army Engineers Waterways Exp. Stat.

Attn: J. P. Holland, A. K. Stagg

3909 Halls Ferry Road

Vicksburg, MS 39180-6199

Dr. G. Zyvoloski

Los Alamos National Laboratory

P. O. Box 1663

Los Alamos, NM 87545

\section{INTERNAL DISTRIBUTION:}

MS

0151 G. Yonas, 9000

0841 P. J. Hommert, 9100

0841 C. Hartwig, 9102

0841 J. H. Biffle, 9103

0828 R. K. Thomas, 9104

0457 J. S. Rottler, 2001

0188 C. E. Meyer, 4523

0827 W. Hermina, 9111

0827 K. S. Chen, 9111

0827 C. E. Hickox, 9111

0827 D. K. Gartling, 9111

0827 S. N. Kempka, 9111

0827 R. P. Schunk, 9111

0834 A. C. Ratzel, 9112

0834 M. R. Baer, 9112

0827 P. L. Hopkins, 9112

350827 M. J. Martinez, 9112

0835 T. Bickel, 9113

0835 R. J. Cochran, 9113

0836 S. R. Tieszen, 9116

0443 H. S. Morgan, 9117

0443 M. C. Stone, 9117

0321 W. Camp, 9200

1111 S. Dosanjh, 9221

1111 G. L. Hennigan, 9221

1111 S. A. Hutchinson, 9221

1111 A. G. Salinger, 9221

1111 J. N. Shadid, 9221

1110 D. Womble, 9222

0819 J. Peery, 9231 
0865 J. Moya, 9735

0724 J. Woodard, 6000

0701 R. W. Lynch, 6100

1033 D. Glowka, 6111

0706 D. Northrop, 6112

0706 J. K. Linn, 6113

1324 P. B. Davies, 6115

1324 R. J. Glass, 6115

1324 C. K. Ho, 6115

1324 S. A. McKenna, 6115

1324 V. C. Tidwell, 6115

1324 S. W. Webb, 6115

0750 M. C. Walck, 6116

1333 L. S. Costin, 6117

0750 H. R. Westrich, 6118

1322 J. R. Tillerson, 6121

0726 J. K. Rice, 6600

0715 R. E. Luna, 6610

0719 G. C. Allen, 6621

0719 J. M. Phelan, 6621

0720 K. Sorenson, 6626

1147 W. Cox, 6681

1147 F. Nimick, 6682

1148 D. Fate, 6685

1395 L. E. Shephard, 6800

1337 F. W. Bingham, 6800

1335 M. S. Chu, 6801

1328 M. G. Marietta, 6821

1320 J. Nowak, 6831

1328 H.-N. Jow, 6848

1328 R. D. Anderson, 6849

1328 M. E. Fewell, 6849

1328 A. H. Treadway, 6849

1328 P. Vaughn, 6849

1326 H. A. Dockery, 6851

1326 J. H. Gauthier, 6851

1326 S. Altman, 6851

1326 M. L. Wilson, 6851

9011 J. Meza, 8950

9042 G. H. Evans, 8345

9042 S. K. Griffiths, 8345

9042 R. H. Nilson, 8345

9018 Central Technical Files, 8523-2

50899 Technical Library, 13414 
0619 Print Media, 12615

20100 Document Processing, 7613-2

For DOE/OSTI 